\title{
Deoxygenation and Other Photochemical Reactions of Aromatic Selenoxides
}

\author{
Ryan D. McCulla and William S. Jenks \\ Iowa State University Ames, IA 50011 \\ wsjenks@iastate.edu
}

Page

S2

S2

S3

S3

S4

S4

S5

S9

S12

S15

S18

S22

S24

S27

\section{Description}

Synthetic details for 1

Synthetic details for 2

Synthetic details for o-Selenocyanobiphenyl

Synthetic details for 2-biphenyl diselenide

Synthetic details for 3

Synthetic details for 5

Spectra for 1

Spectra for 2

Spectra for $o$-Selenocyanobiphenyl

Spectra for 2-biphenyl diselenide

Spectra for 3

Spectra for 5

Spectra for 8

Spectra for 9 
Dibenzoselenophene oxide (1). ${ }^{31}$ Dibenzoselenophene $(0.317 \mathrm{~g}, 1.2 \mathrm{mmol})$ was dissolved in $50 \mathrm{~mL}$ dichloromethane and cooled to $-78{ }^{\circ} \mathrm{C} . \quad m \mathrm{CPBA}(1.4 \mathrm{mmol}$, as the commercial mixture, dissolved in $10 \mathrm{~mL} \mathrm{CH}_{2} \mathrm{Cl}_{2}$ ) was added dropwise. The mixture was stirred while it was allowed to slowly warm to $0{ }^{\circ} \mathrm{C}$. The reaction mixture was then added to saturated aqueous $\mathrm{NaHCO}_{3}$. The organic layer was washed with water, dried with anhydrous $\mathrm{MgSO}_{4}$, and then concentrated under reduced pressure. This produced $0.188 \mathrm{~g}$ of a white crystal. The crystals were purified by multiple careful recrystallizations from $\mathrm{CH}_{2} \mathrm{Cl}_{2}$ to produce $0.150 \mathrm{~g}$ of a white crystal, $\mathbf{1}$ (46\% yield). ${ }^{1} \mathrm{H}$ NMR (300 MHz, CDCl $)$ d $7.96(2 \mathrm{H}, \mathrm{d}, \mathrm{J}=7.5 \mathrm{~Hz}), 7.83(2 \mathrm{H}, \mathrm{d}, \mathrm{J}=7.2 \mathrm{~Hz}), 7.63(2 \mathrm{H}$, $\mathrm{td}, \mathrm{J}=7.5 \mathrm{~Hz}, \mathrm{~J}=1.2 \mathrm{~Hz}), 7.51(2 \mathrm{H}, \mathrm{J}=7.5 \mathrm{~Hz}, \mathrm{~J}=1.2 \mathrm{~Hz}) ;{ }^{13} \mathrm{C} \mathrm{NMR}\left(75 \mathrm{MHz}, \mathrm{CDCl}_{3}\right)$ д 145.3, 141.0, 132.9, 130.3, 129.3, 123.4; GCMS (EI) m/z 247, 231, 220, 168, 152, 139.

Phenyl Selenoxide (2). ${ }^{54}$ m-CPBA $(2.00 \mathrm{~g}, 8.2 \mathrm{mmol})$ was dissolved in $50 \mathrm{~mL}$ dichloromethane and cooled to $-78{ }^{\circ} \mathrm{C}$. To this was added diphenyl selenide $(1.5 \mathrm{~mL}, 8.6$ mmol). The mixture was stirred for $10 \mathrm{~min}$, then poured onto saturated $\mathrm{NaHCO}_{3}$ and dichloromethane. The organic layer was washed thrice with water and then dried with anhydrous $\mathrm{MgSO}_{4}$. The solvent was removed under reduce pressure to produce $1.9 \mathrm{~g}$ crude orange solid. This was recrystallized from a 3:1 hexane:dichloromethane solution to produce phenyl selenoxide (0.33 g, $1.3 \mathrm{mmol})$, as a white solid (16\%). ${ }^{1} \mathrm{H}$ NMR (400 $\left.\mathrm{MHz}, \mathrm{CDCl}_{3}\right)$ d $7.615(4 \mathrm{H}, \mathrm{m}), 7.339(6 \mathrm{H}, \mathrm{m}) ;{ }^{13} \mathrm{C} \mathrm{NMR}\left(100 \mathrm{MHz}, \mathrm{CDCl}_{3}\right)$ д 142.78, 131.17, 129.58, 126.10; GCMS (EI) $m / z$ 250, 170, 157, 151, 135, 77.

The preparation of dibenzoselenophene (3) from $o$-aminobiphenyl was based on the method of McCullough, ${ }^{31}$ with some modification. The spectral data reported below are new. 
o-Selenocyanobiphenyl. A mixture of $5.0 \mathrm{~mL}$ concentrated sulfuric acid and $20 \mathrm{~mL}$ of water were warmed. $o$-Aminobiphenyl $(4.98 \mathrm{~g}, 29.4 \mathrm{mmol})$ was dissolved in the acidic solution, which was cooled down to $0{ }^{\circ} \mathrm{C}$ before $80 \mathrm{~mL}$ of water was added. With stirring, $\mathrm{NaNO}_{2}(2.5 \mathrm{~g}, 36.2 \mathrm{mmol})$ was added. Care was taken to ensure that the reaction mixture was kept below $7{ }^{\circ} \mathrm{C}$. Sodium acetate was then added to the brown solution until it turned a dirty yellow when the $\mathrm{pH}$ was 6.0. Potassium selenocyanate $(4.2 \mathrm{~g}, 29.1$ mmol) was dissolved in $20 \mathrm{~mL}$ of water and then added to the reaction mixture slowly. This created a frothy orange solution, which was allowed to evolve for little over an hour. The mixture was then poured onto $70 \mathrm{~mL}$ toluene. The organic layer was washed twice with water and then dried with anhydrous $\mathrm{MgSO}_{4}$. The organic was run through a silica plug to remove salts. Toluene was then removed under reduced pressure to yield a dark orange oil. Distillation at $175{ }^{\circ} \mathrm{C}$ and 2 Torr yields $3.7 \mathrm{~g}$ (49\%) of $98 \%$ $o$-selenocyanobiphenyl, which is an oil. Further purification can be performed by preparatory thin layer chromatography on silica (eluent: 3:1 Hexanes:Ethyl Acetate, $\mathrm{R}_{f}=$ .68): IR (NaCl) $v_{\max } 3057,3026,2153 \mathrm{~cm}^{-1} ;{ }^{1} \mathrm{H}$ NMR $\left(300 \mathrm{MHz}, \mathrm{CDCl}_{3}\right)$ d 7.85-7.80 (1H, m), 7.50-7.40 (5H, m), 7.33-7.24 (3H, m); ${ }^{13} \mathrm{C}$ NMR (300 MHz, $\left.\mathrm{CDCl}_{3}\right)$ д 142.8, 140.0, 130.9, 130.8, 129.2, 129.1, 129.0, 128.7, 128.1, 123.9, 102.4; GCMS (EI) $m / z$ 259, 232, $196,179$.

2-biphenyl diselenide. Potassium hydroxide $(0.78 \mathrm{~g}, 13.9 \mathrm{mmol})$ was dissolved in 4 $\mathrm{mL}$ methanol. Then $1.06 \mathrm{~g}(4.1 \mathrm{mmol})$ of the $o$-selenocyanobiphenyl oil was added, forming an orange-yellow precipitate. This mixture was then vigorously swirled, and then allowed to stand for $30 \mathrm{~min}$. The reaction mixture was then poured onto toluene and saturated aqueous boric acid solution. The organic was washed twice with water, dried 
with anhydrous $\mathrm{MgSO}_{4}$, and removed under reduced pressure to produce an orange oil. The oil was dissolved in acetone and cooled to produce yellow crystals. The crystals were then recrystallized twice from acetone to produce 2-biphenyl diselenide (1.05 g, 2.2 mmol, 55\% yield): IR (NaCl) $v_{\max } 3080,3057,3024$; GCMS (EI) $m / z, 466,232,152 ;{ }^{1} \mathrm{H}$ NMR $\left(400 \mathrm{MHz}, \mathrm{CDCl}_{3}\right)$ d 7.69-7.66 $(1 \mathrm{H}, \mathrm{m}), 7.50-7.36(5 \mathrm{H}, \mathrm{m}) 7.28-7.18(3 \mathrm{H}, \mathrm{m}) ;{ }^{13} \mathrm{C}$ NMR (400 MHz, $\left.\mathrm{CDCl}_{3}\right)$ 142.6, 141.3, 130.8, 130.0, 129.9, 129.4 128.8, 128.2, 127.2.

Dibenzoselenophene (3). Biphenyl diselenide (1.13 g, $2.4 \mathrm{mmol})$ was dissolved in 15 $\mathrm{mL}$ carbon tetrachloride. With stirring, the solution was heated to $100{ }^{\circ} \mathrm{C}$ and bromine $(0.10 \mathrm{~mL} 2.0 \mathrm{mmol})$ was added. After four h, the bath temperature was lowered to $65{ }^{\circ} \mathrm{C}$ for another $18 \mathrm{~h}$. The solvent was removed under reduced pressure. The resulting material was purified by flash chromatography (eluent: hexane $\mathrm{R}_{f}=0.38$ ) This produced $0.745 \mathrm{~g}$ of $99 \%$ pure dibenzoselenophene ( $81 \%$ yield). ${ }^{1} \mathrm{H}$ NMR $\left(300 \mathrm{MHz}, \mathrm{CDCl}_{3}\right) \partial$ $8.17(2 \mathrm{H}, \mathrm{dq} \mathrm{J}=7.8 \mathrm{~Hz}, \mathrm{~J}=0.6 \mathrm{~Hz}), 7.89(2 \mathrm{H}, \mathrm{dq}, \mathrm{J}=7.8 \mathrm{~Hz}, \mathrm{~J}=0.6 \mathrm{~Hz}), 7.54(2 \mathrm{H}, \mathrm{td}, \mathrm{J}$ $=7.8 \mathrm{~Hz}, \mathrm{~J}=1.2 \mathrm{~Hz}), 7.45(2 \mathrm{H}, \mathrm{td}, \mathrm{J}=7.8 \mathrm{~Hz}, \mathrm{~J}=1.2 \mathrm{~Hz}) .{ }^{13} \mathrm{C} \mathrm{NMR}\left(75 \mathrm{MHz}, \mathrm{CDCl}_{3}\right) \partial$ $139.6,138.6127 .2,126.4,125.2,123.2$.

Dibenzoselenophene-Se-Se-dioxide (5). ${ }^{55}$ A $50 \mathrm{~mL}$ round bottom flask was charged with dibenzoselenophene (26.4 mg, $0.11 \mathrm{mmol})$ and $2 \mathrm{~mL}$ dichloromethane.

Dimethyldioxirane in acetone ${ }^{30}(5 \mathrm{~mL}, 0.08 \mathrm{M}, 4 \mathrm{mmol})$ was added. After standing overnight, the solvent was removed under reduced pressure to leave behind dibenzoselenophene-Se-Se-dioxide (10 mg, 50\%). ${ }^{1} \mathrm{H}$ NMR (300 MHz, $\mathrm{CDCl}_{3}$ ) д 8.02 $(2 \mathrm{H}, \mathrm{dd}, \mathrm{J}=7.2 \mathrm{~Hz}, \mathrm{~J}=0.6 \mathrm{~Hz}), 7.97(2 \mathrm{H}, \mathrm{td}, \mathrm{J}=7.8 \mathrm{~Hz}, \mathrm{~J}=0.6 \mathrm{~Hz}), 7.79(2 \mathrm{H}, \mathrm{td}, \mathrm{J}=$ $7.5 \mathrm{~Hz}, \mathrm{~J}=1.2 \mathrm{~Hz}), 7.68(2 \mathrm{H}, \mathrm{td}, \mathrm{J}=7.5 \mathrm{~Hz}, \mathrm{~J}=1.2 \mathrm{~Hz}) ;{ }^{13} \mathrm{C} \mathrm{NMR}\left(75 \mathrm{MHz}, \mathrm{CDCl}_{3}\right) \partial$ 
136.76, 135.31, 132.57, 131.92, 125.95, 123.25; GCMS (EI) $m / z$ 264, 235, 233, 168, 152 , $139,128$.

\section{Compound 1}

Dibenzoselenophene-Se-oxide

${ }^{1} \mathrm{H}$ NMR spectrum for $\mathbf{1}$

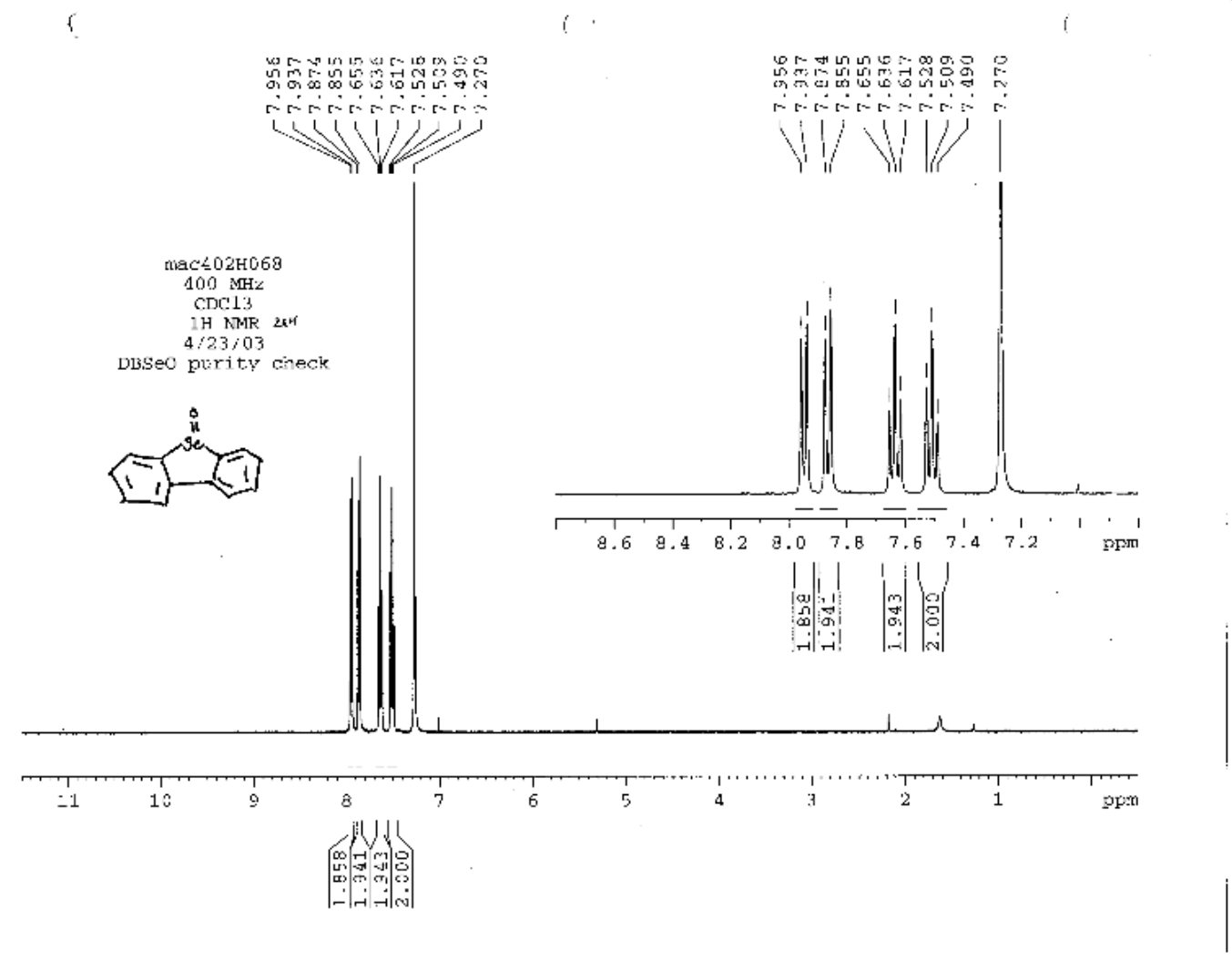


${ }^{13} \mathrm{C}$ NMR spectrum for $\mathbf{1}$

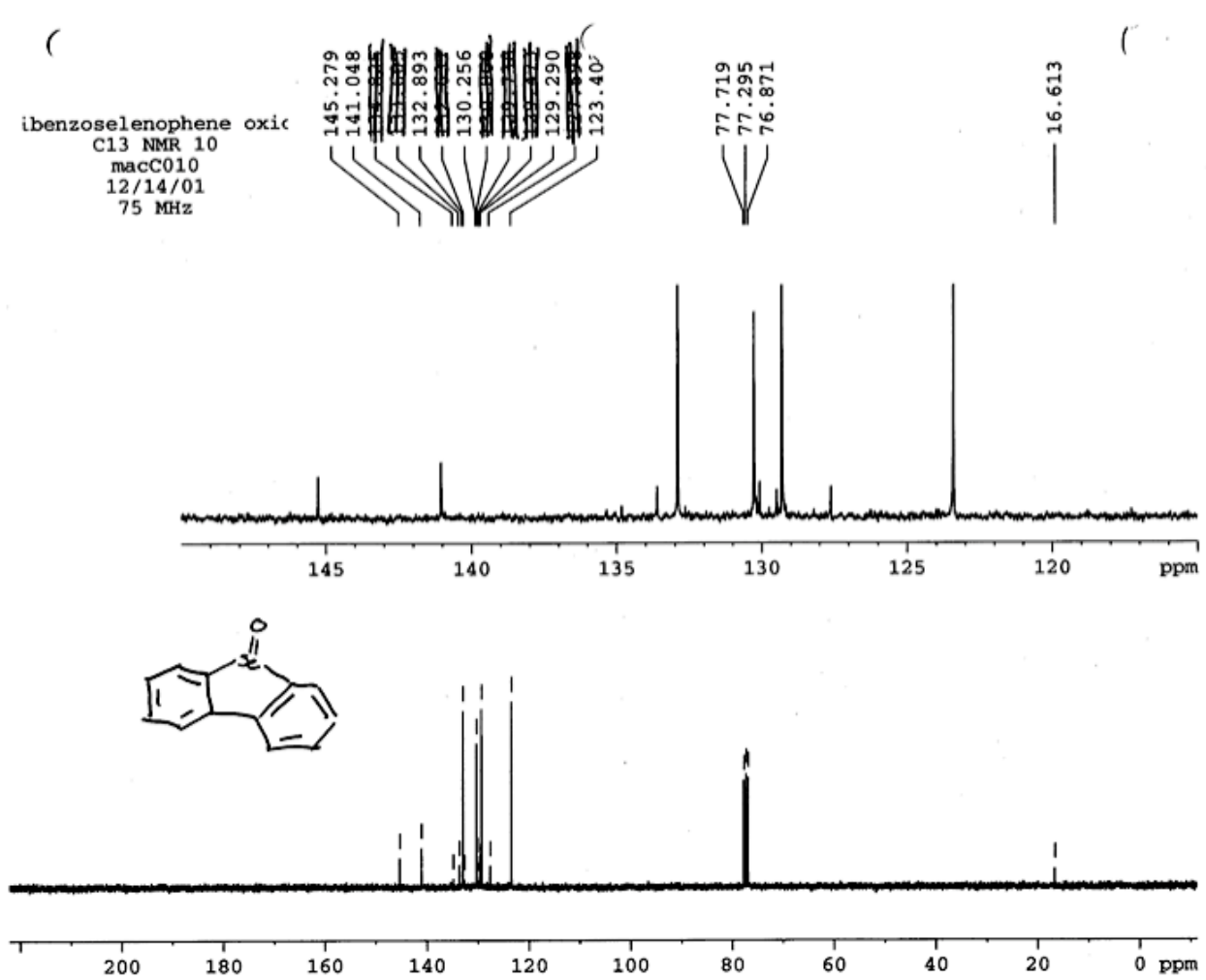




\section{MS spectrum for $\mathbf{1}$}

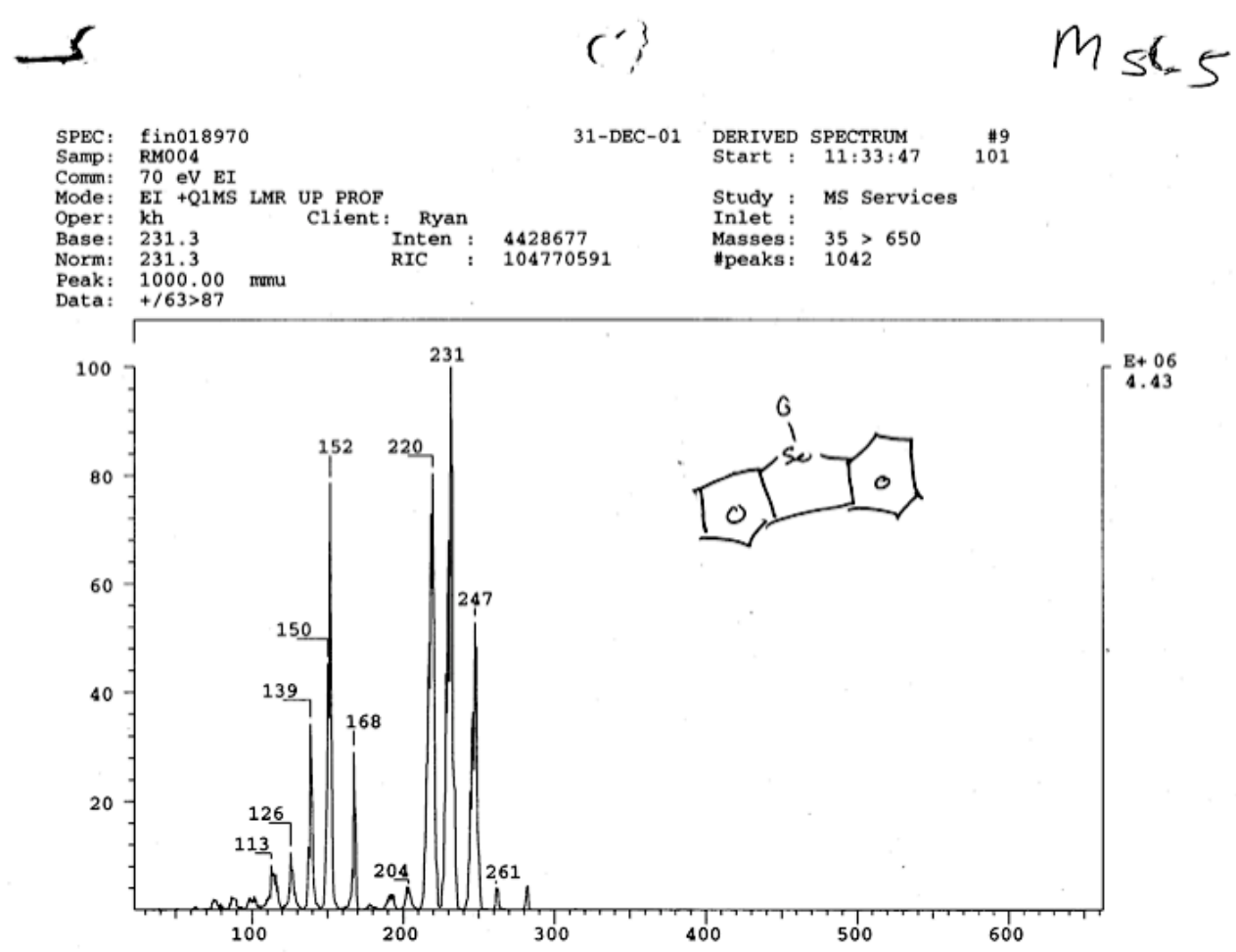


UV spectrum for $\mathbf{1}$

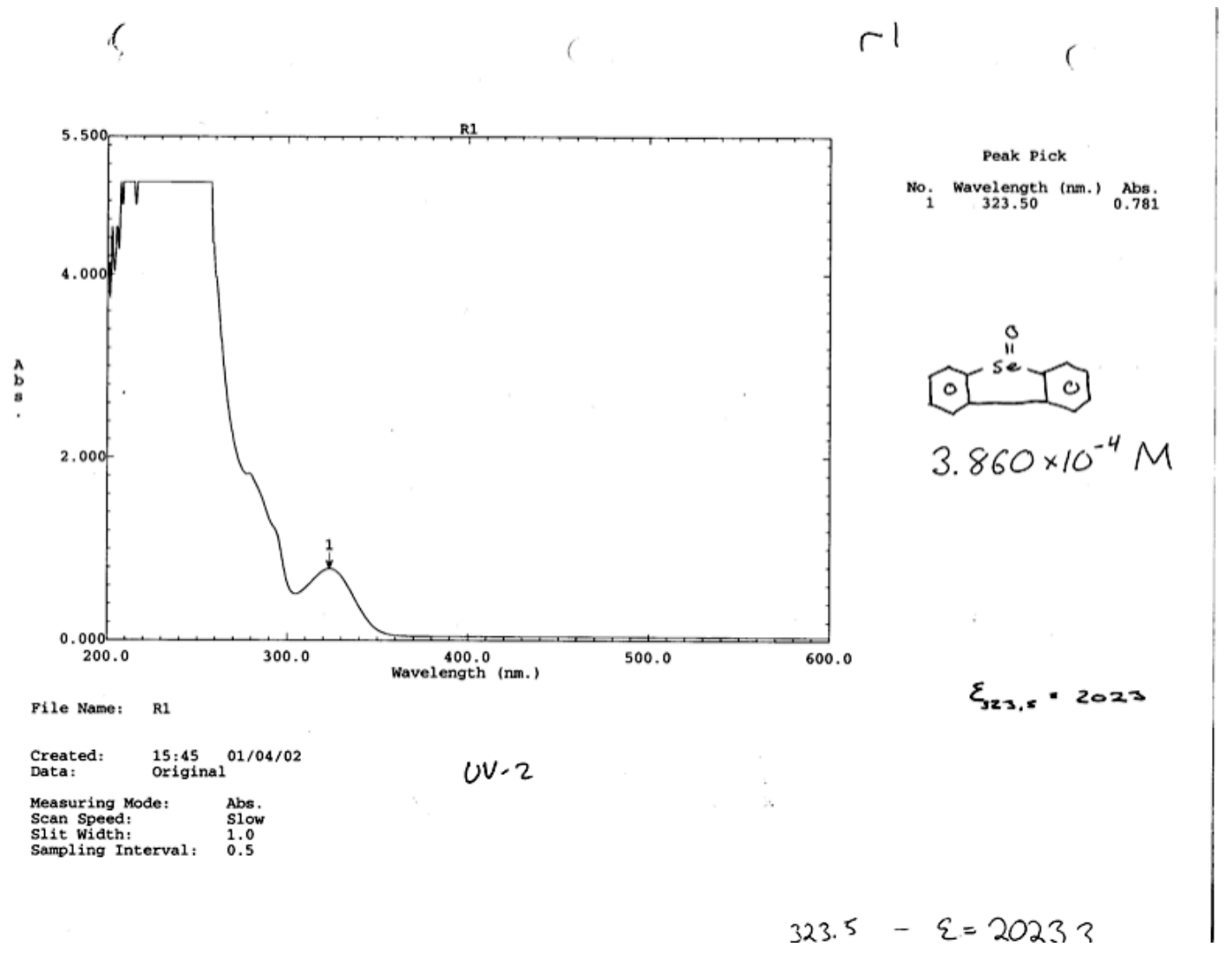


Compound 2

Diphenylselenoxide-Se-oxide

${ }^{1} \mathrm{H}$ NMR spectrum for 2

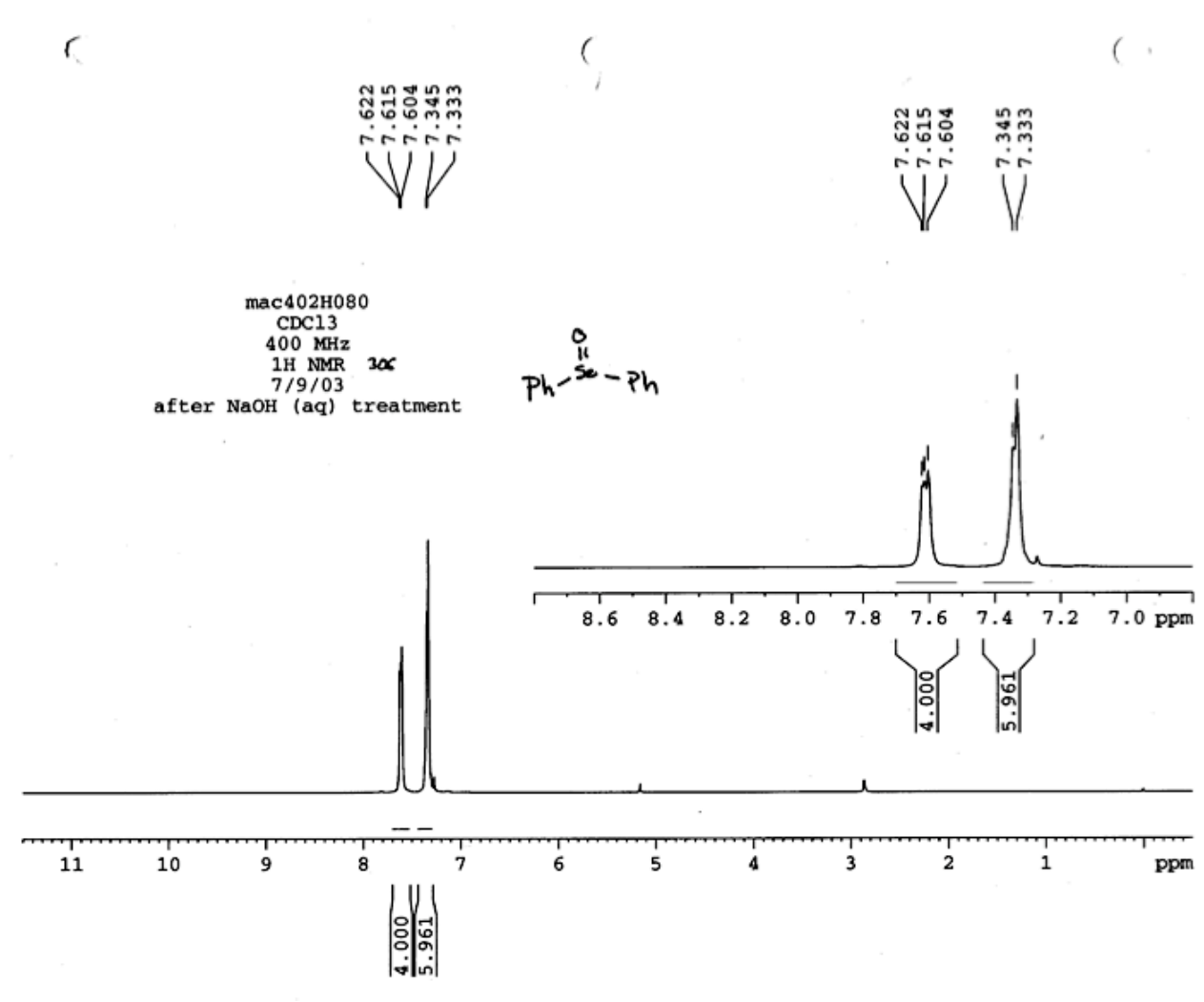


${ }^{13} \mathrm{C}$ NMR spectrum for 2

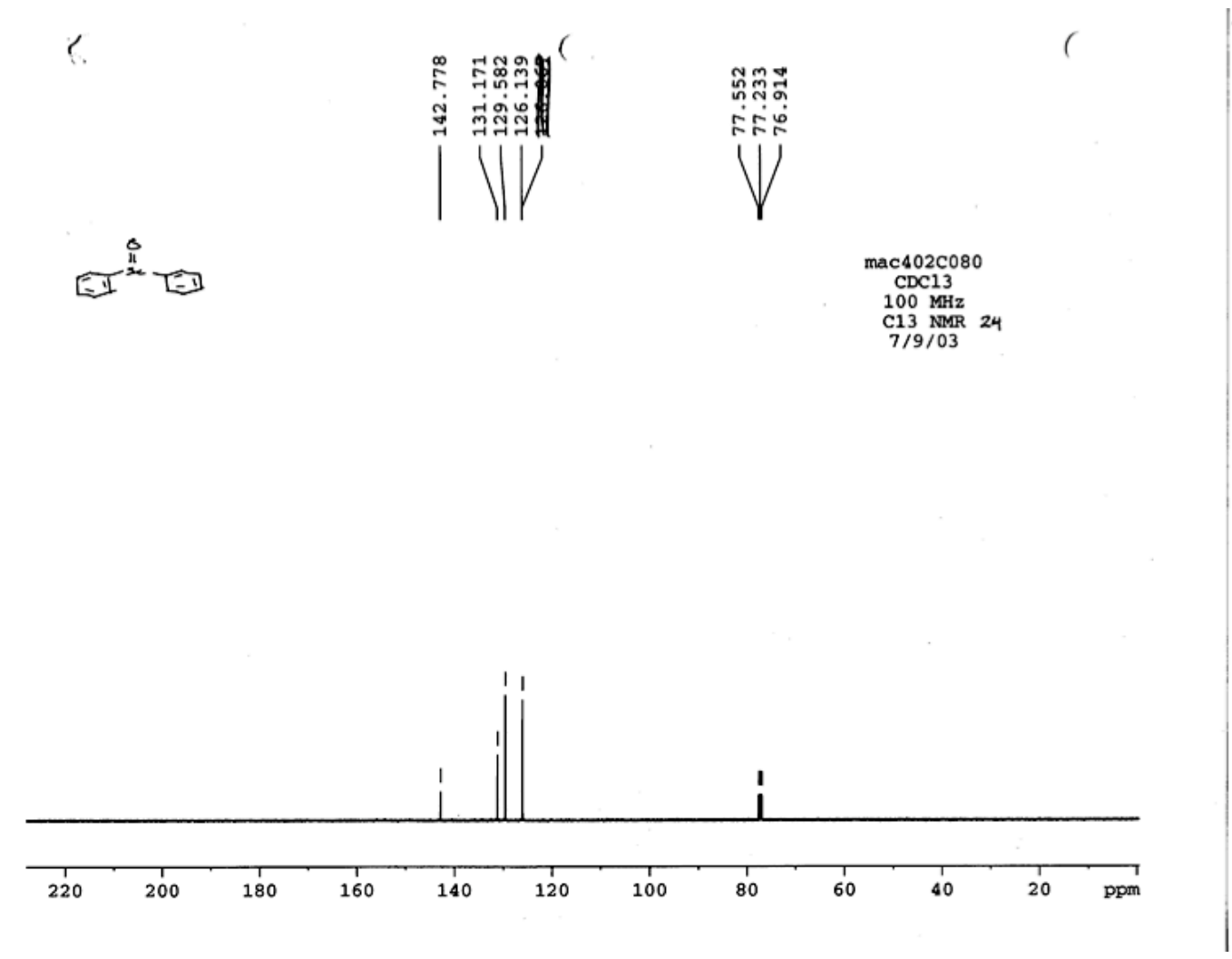




\section{MS spectrum for $\mathbf{2}$}

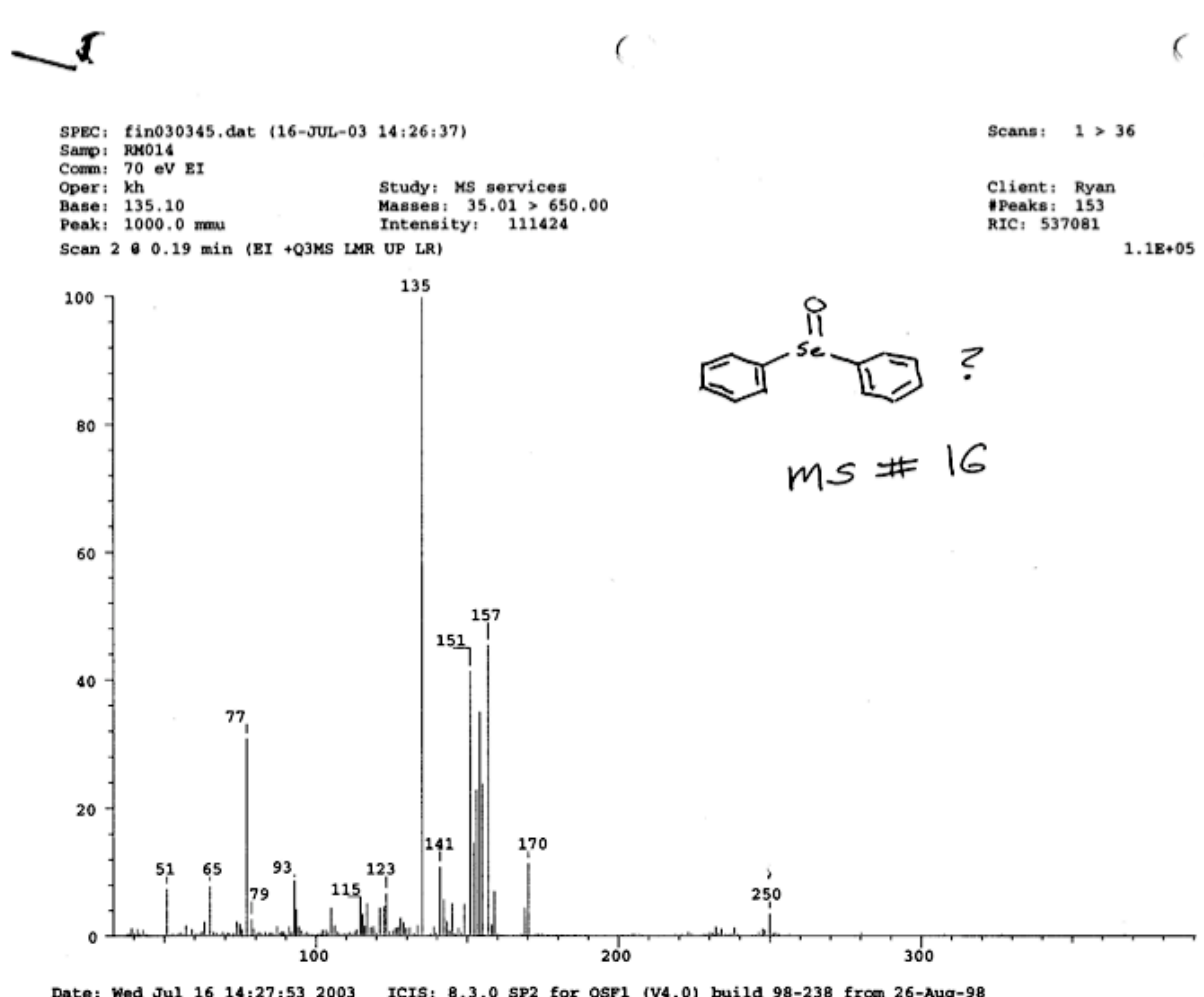

UV spectrum for $\mathbf{2}$
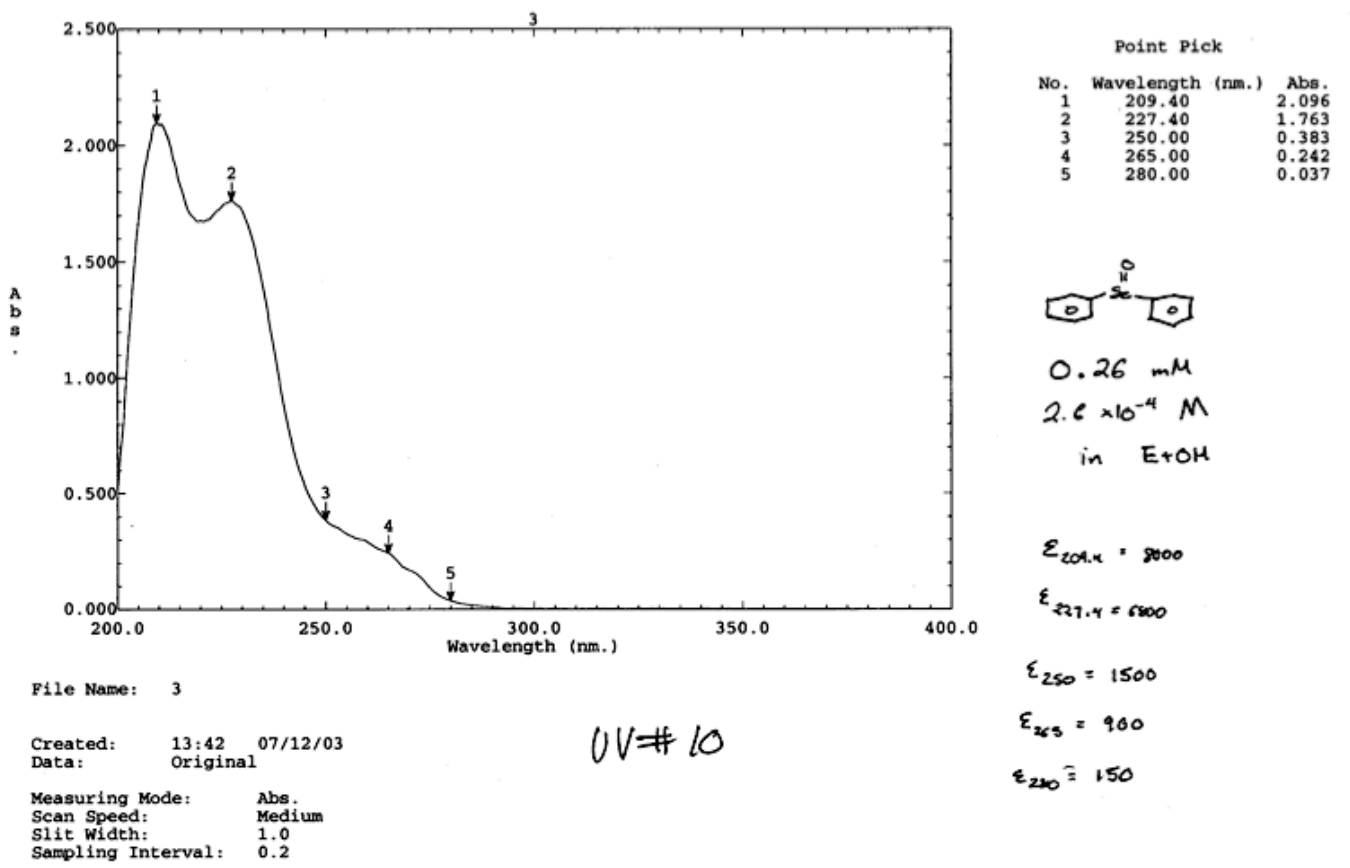

$$
\begin{aligned}
& \varepsilon_{200.4}=8000 \\
& \varepsilon_{227.4}=0000 \\
& \varepsilon_{250}=1500 \\
& \varepsilon_{265}=900 \\
& \varepsilon_{250}=150
\end{aligned}
$$


$o$-Selenocyanobiphenyl

${ }^{1} \mathrm{H}$ NMR spectrum for $o$-Selenocyanobiphenyl
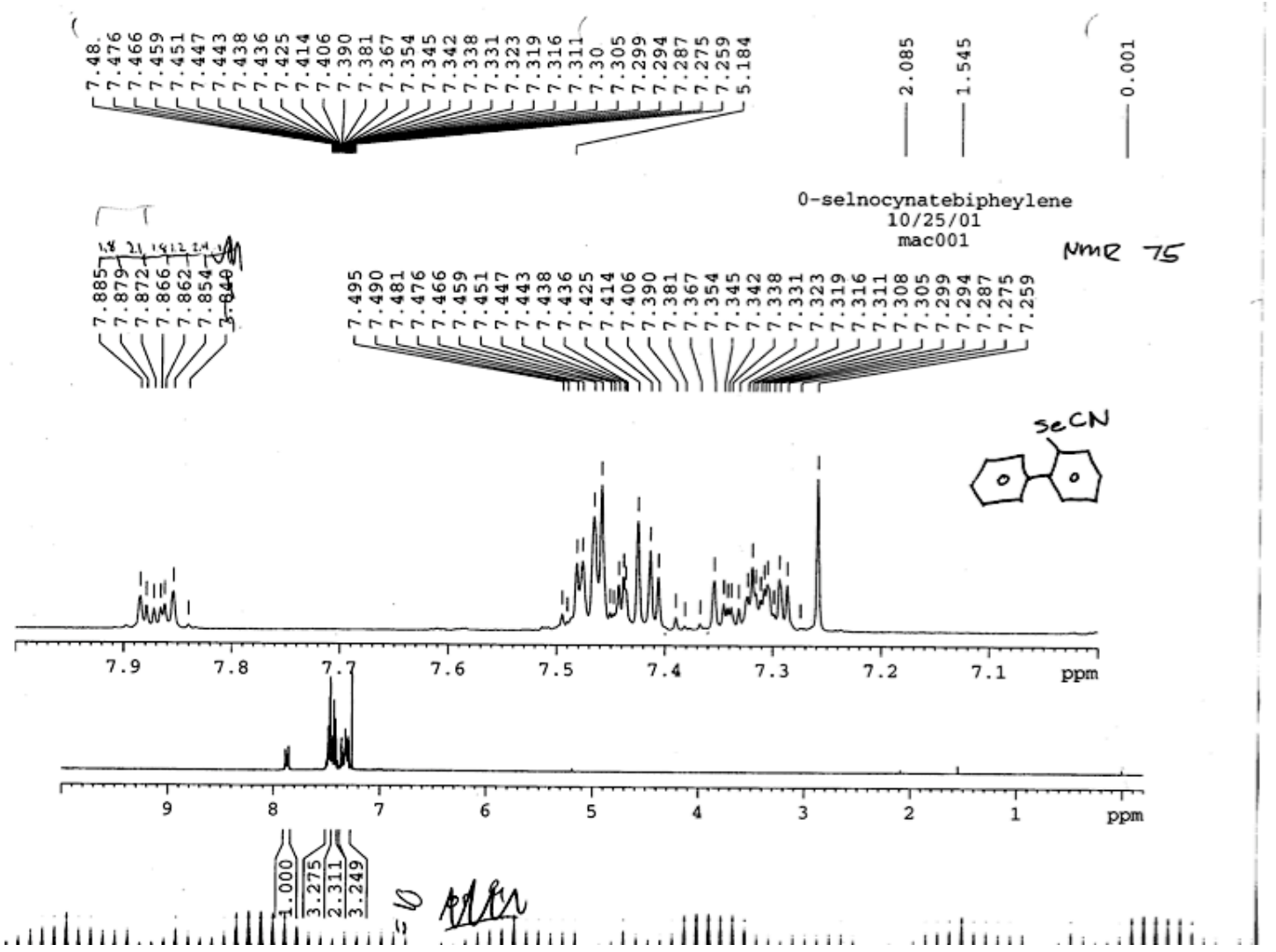
${ }^{13} \mathrm{C}$ NMR spectrum for $o$-Selenocyanobiphenyl
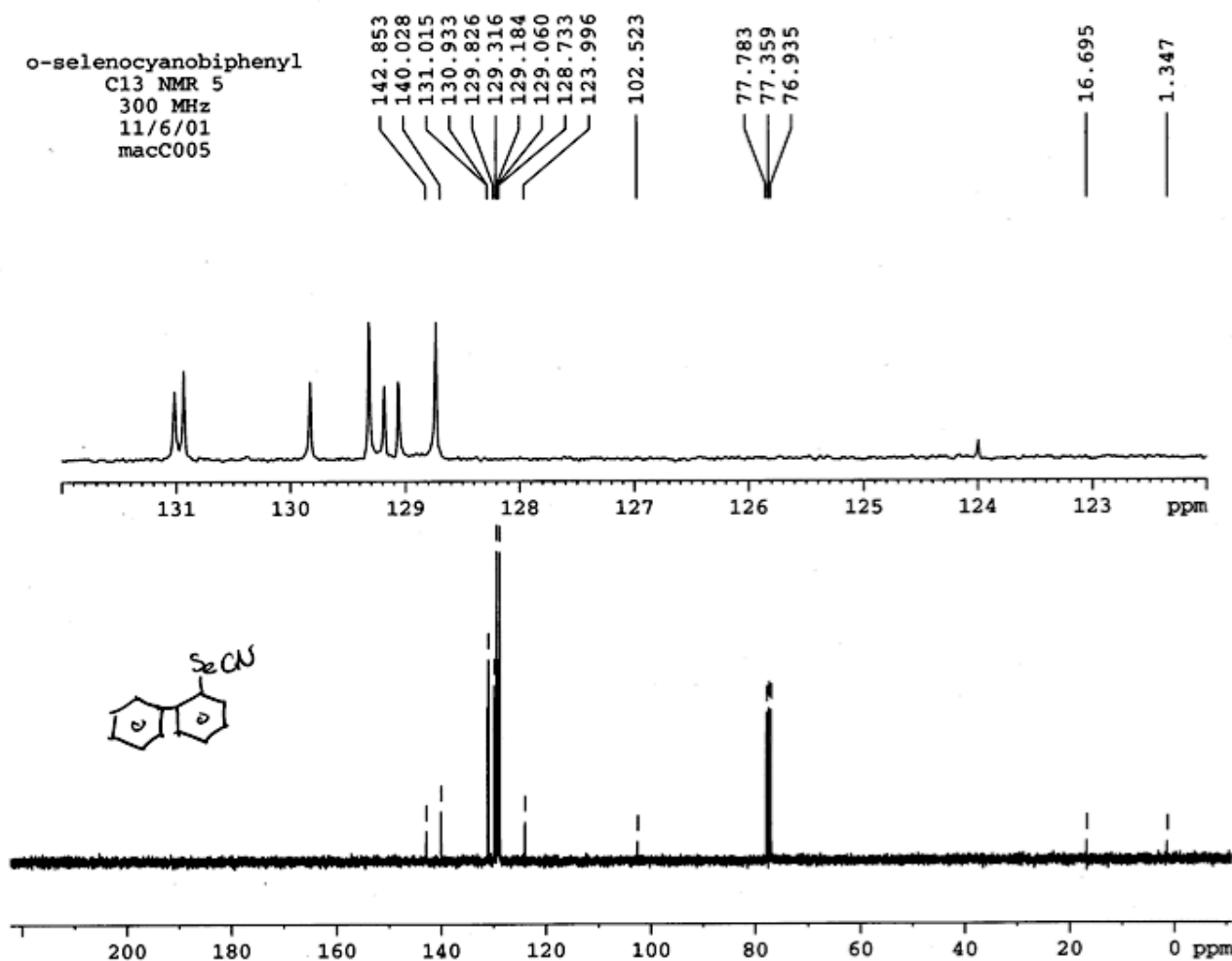

MS spectrum for $o$-Selenocyanobiphenyl

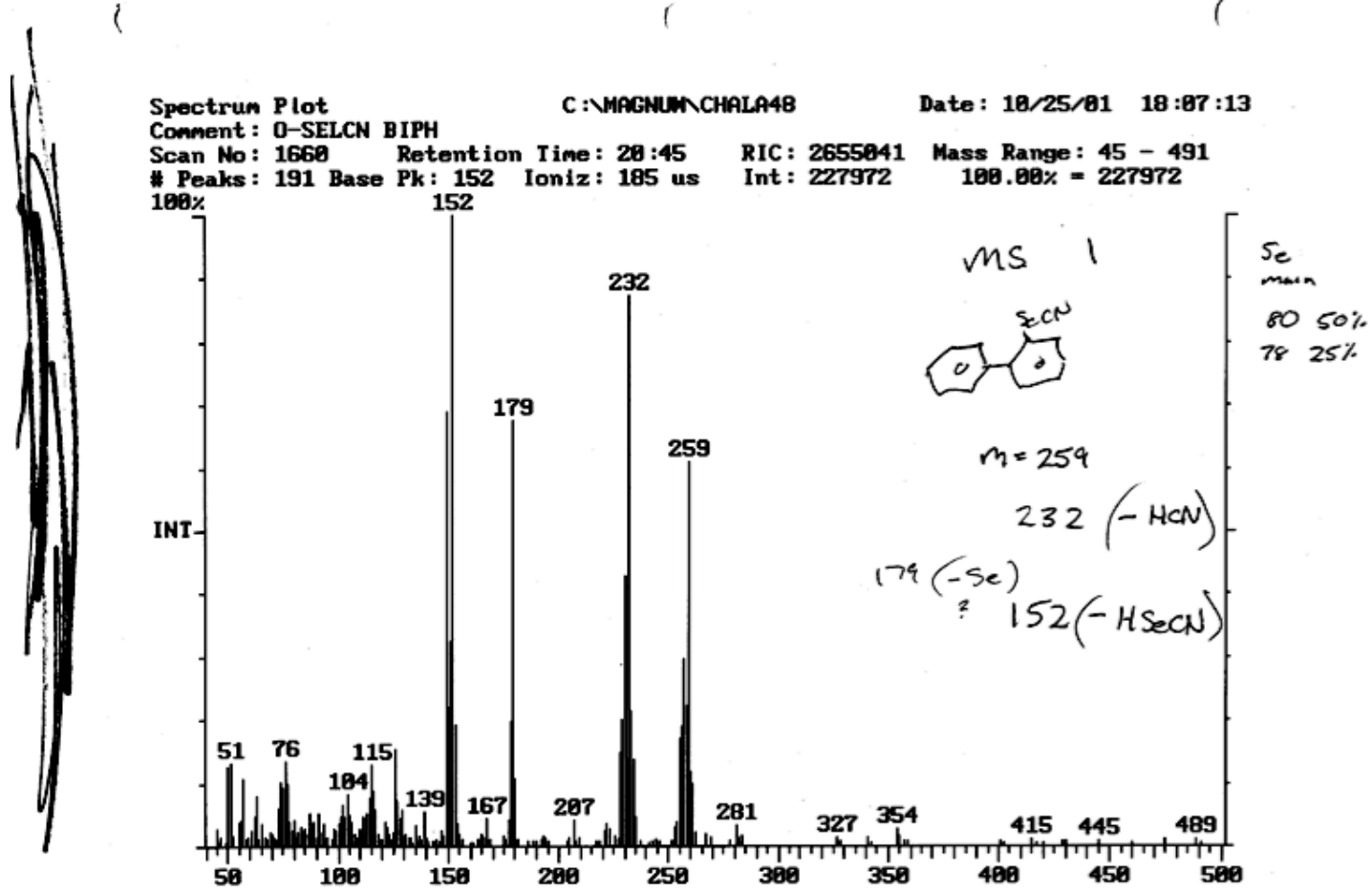


IR spectrum for $o$-Selenocyanobiphenyl

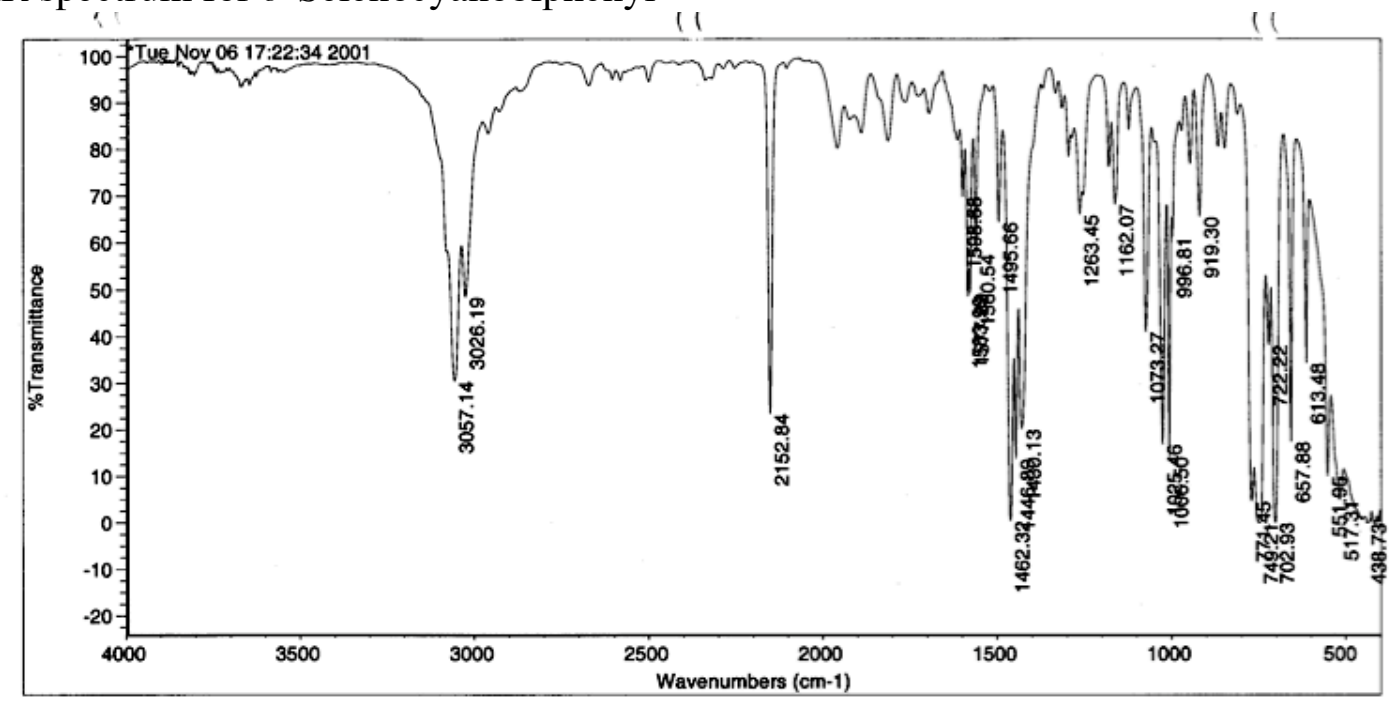

Tue Nov 06 17:23:23 2001

FIND PEAKS:

Spectrum:

Region:

'Tue Nov 06 17:22:34 2001

$4000.00 \quad 400.00$

Absolute threshold: 70.721

Sensitivity:

50

Position: $\mathbf{7 4 9 . 2 1}$ Intensity: -0.0073

Position: 438.73 Intensity: -0.143

Position: 702.93 Intensity: 0.0089

Position: 1462.32 Intensity: 0.417

Position: 771.45 Intensity: 4.648

Position: $\quad 517.31$ Intensity: 4.944

Position: 551.05 Intonsity: 10.040

Position: 551.95 Intons: 10.040

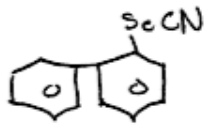

IR \# 
2-Biphenyl Diselenide

${ }^{1} \mathrm{H}$ NMR spectrum for 2-Biphenyl Diselenide

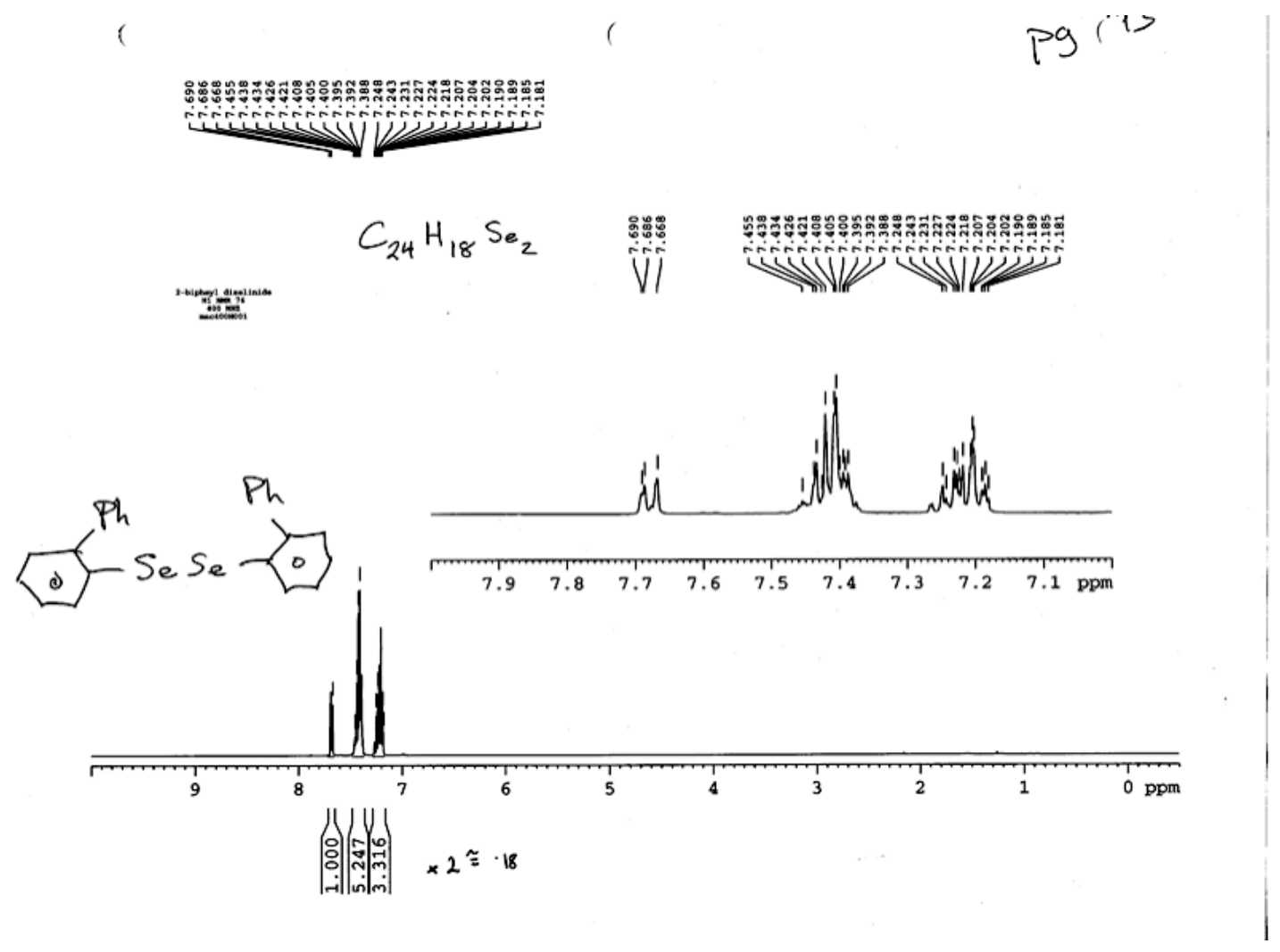


${ }^{13} \mathrm{C}$ NMR spectrum for 2-Biphenyl Diselenide

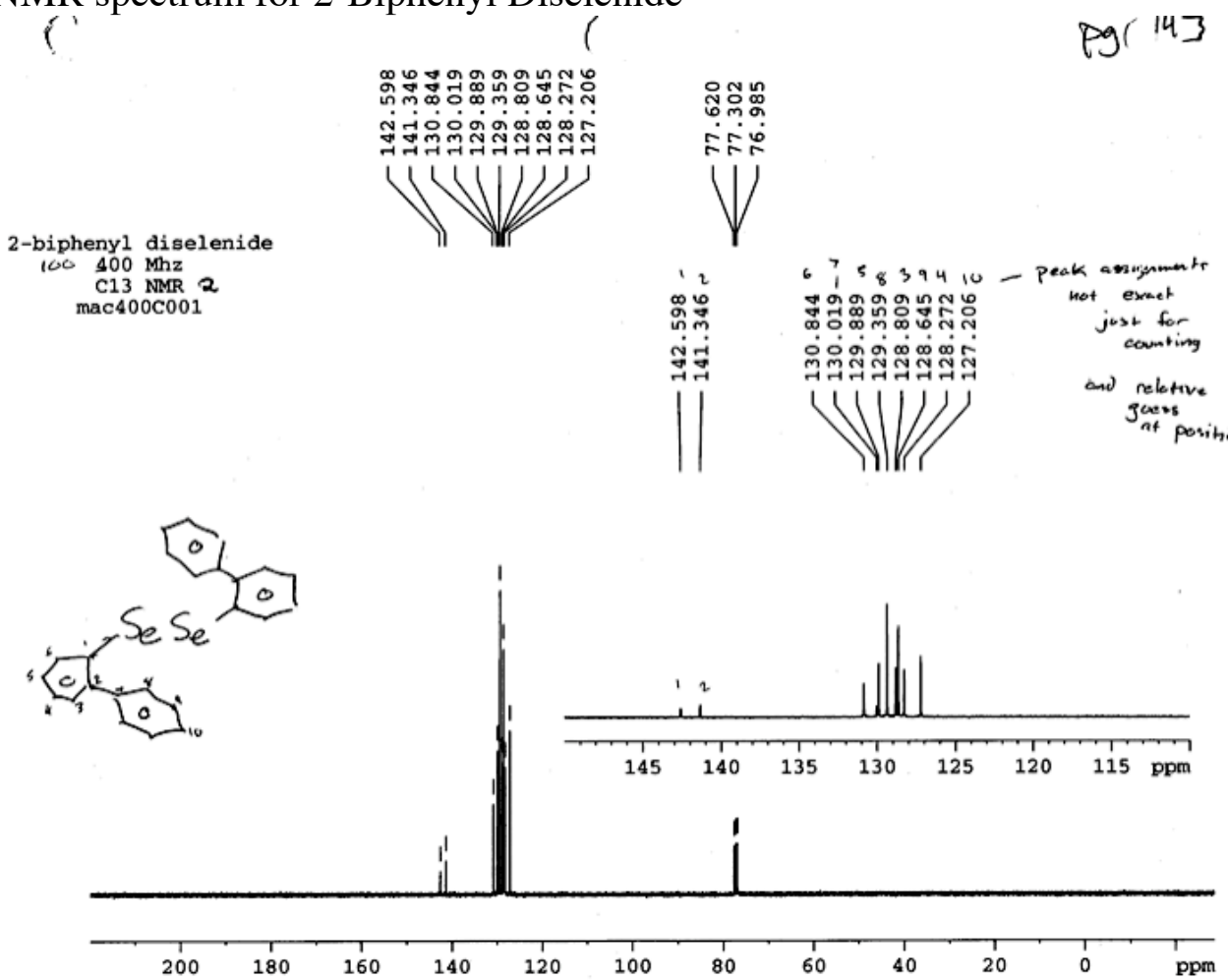

MS spectrum for 2-Biphenyl Diselenide

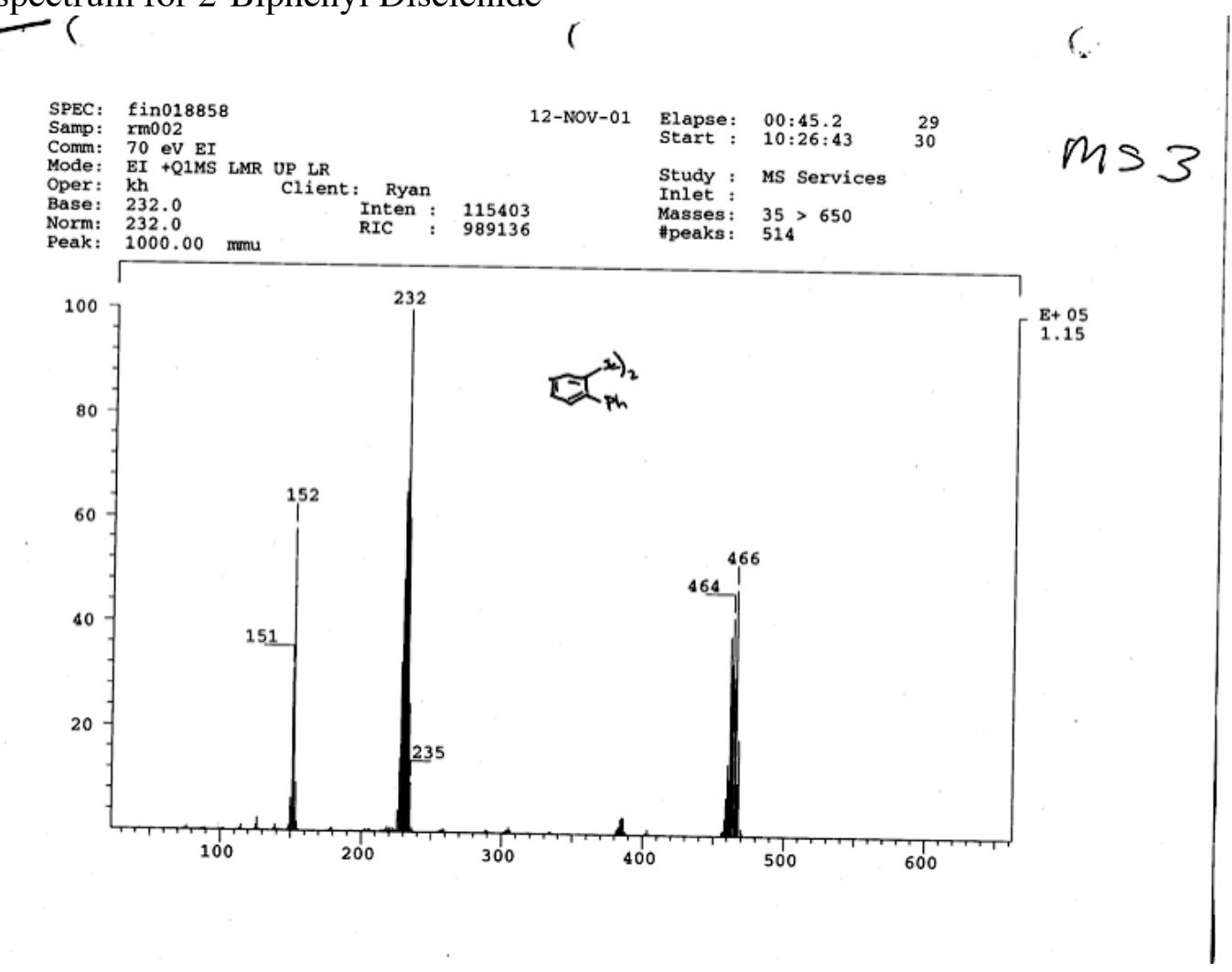


IR spectrum for 2-Biphenyl Diselenide

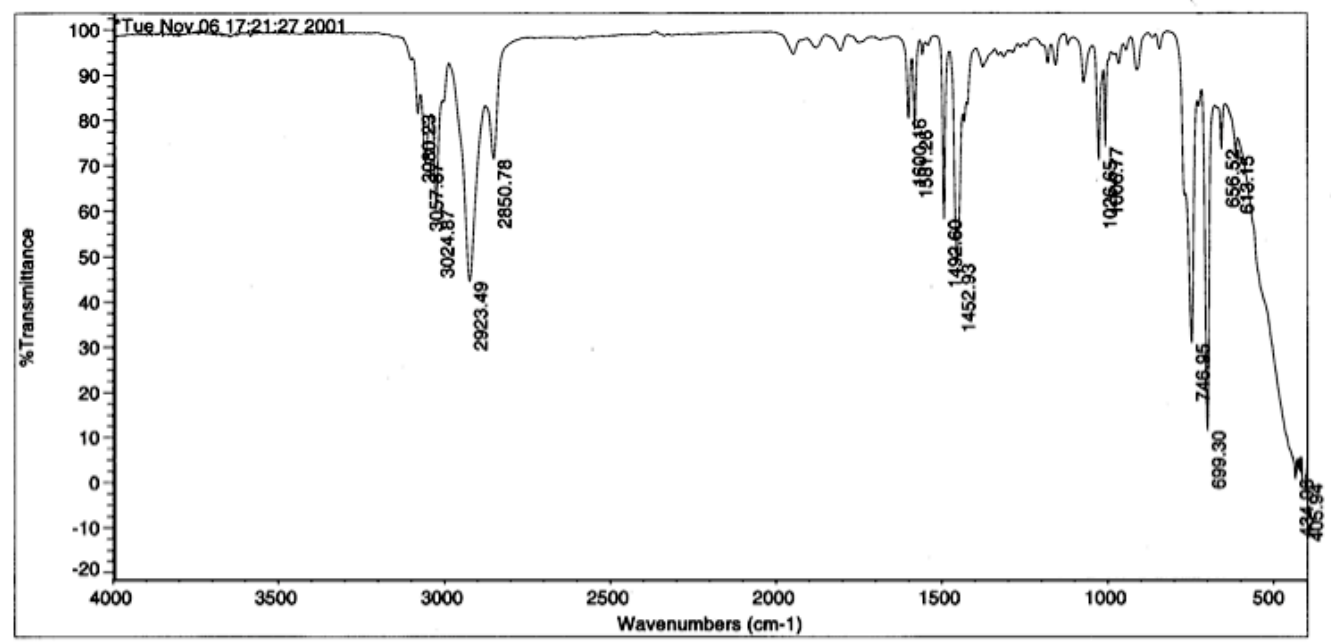

Tue Nov 06 17:21:56 2001

FIND PEAKS:

Spectrum:

Region:

Absolute thre

-Tue Nov 06 17:21:27 2001

Sensitivity:

4000.00

Postion:

Position: 405.94 Intensity: -0.219

Position: 434.96 Intensity: 0.809

Position: $\quad 699.30$ Intensity: 11.459

Position: 746.95 Intensity: 30.888

Position: 2923.49 Intensily: $\mathbf{4 4 . 4 8 2}$

Position: 1492.60 Intensity: 58.192

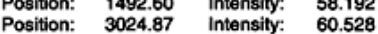

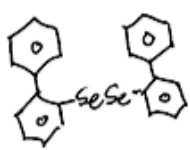

IR\#2 
Compound $\mathbf{3}$

Dibenzoselenophene

${ }^{1} \mathrm{H}$ NMR spectrum for $\mathbf{3}$

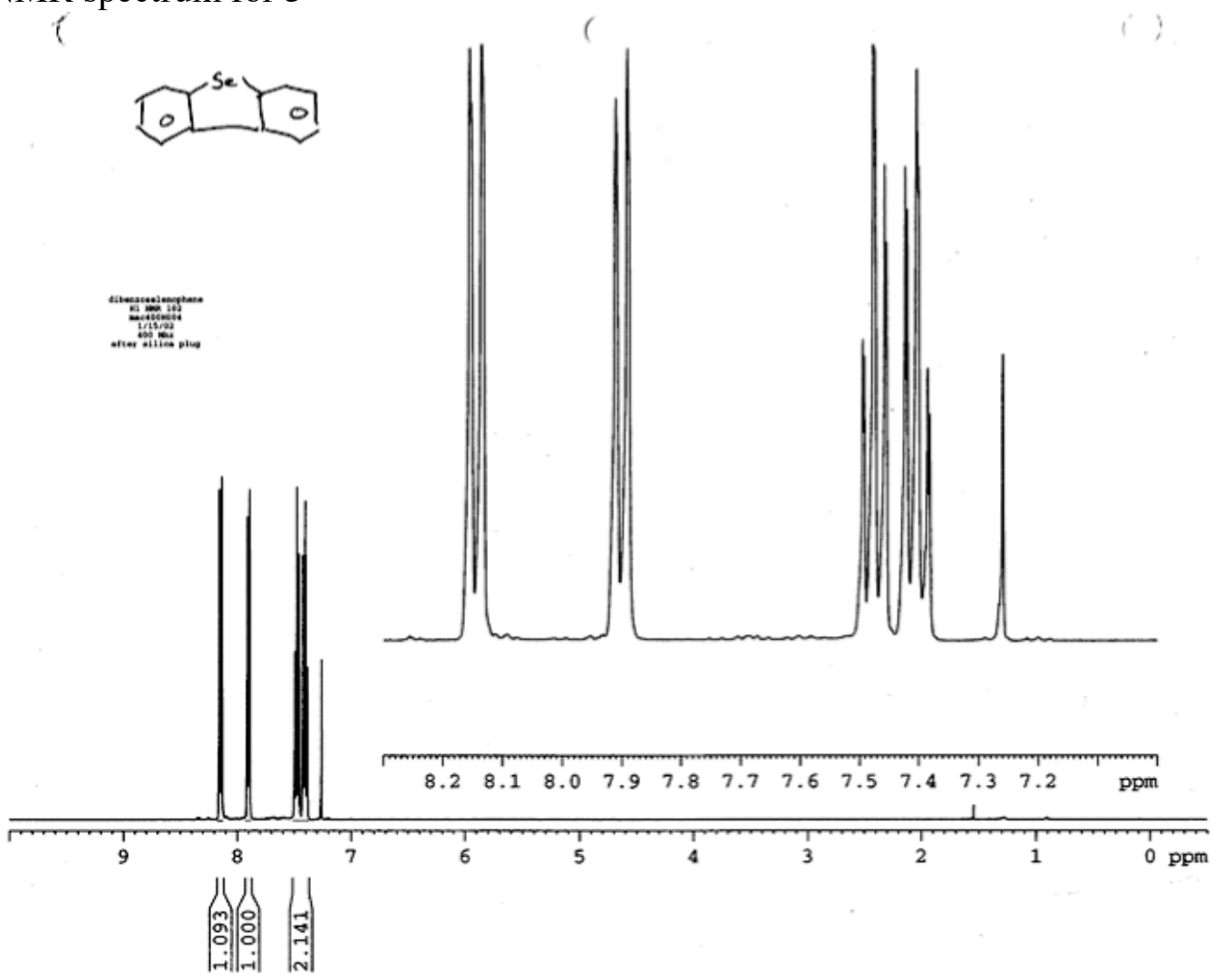


${ }^{13} \mathrm{C}$ NMR spectrum for $\mathbf{3}$
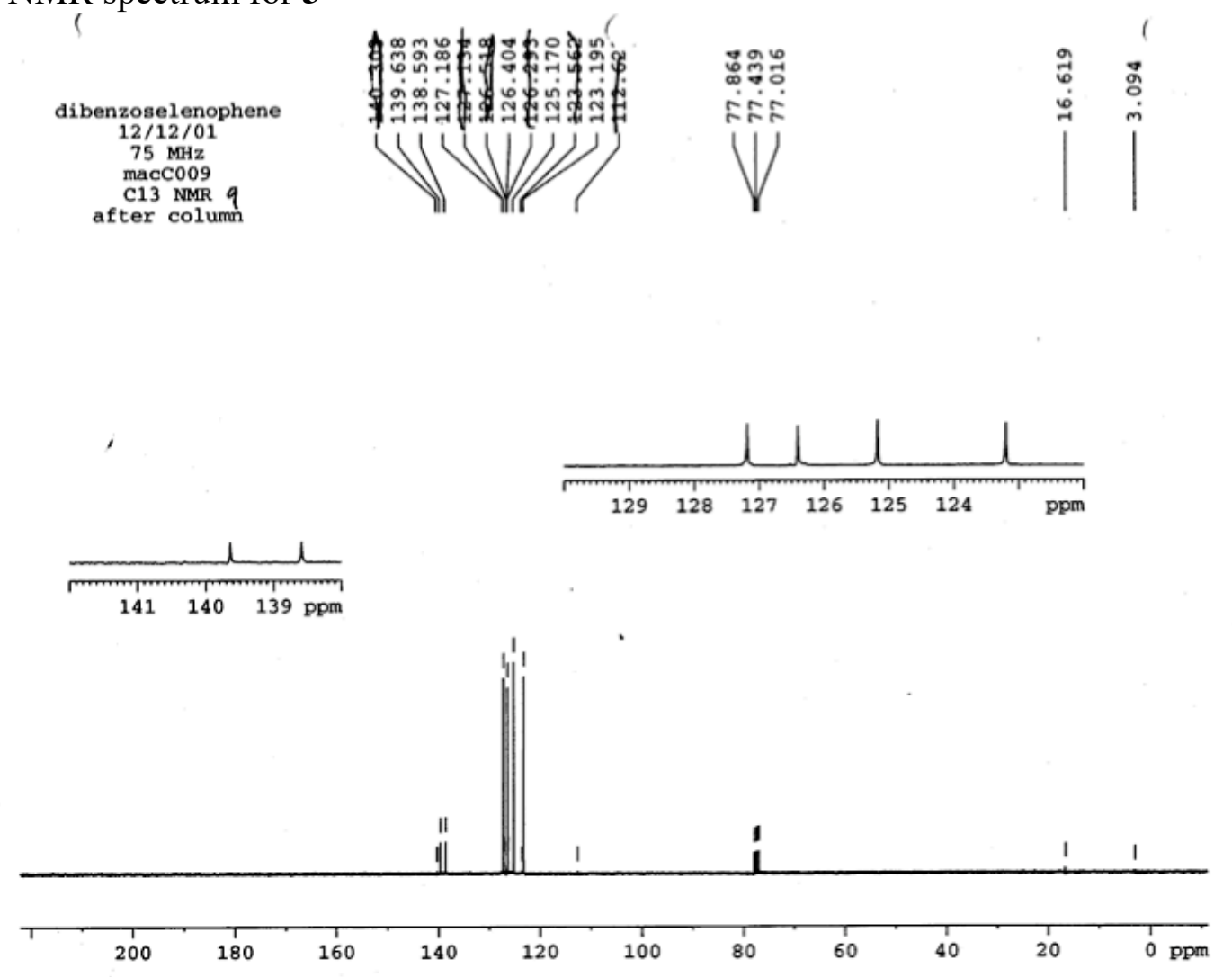
MS spectrum for 3

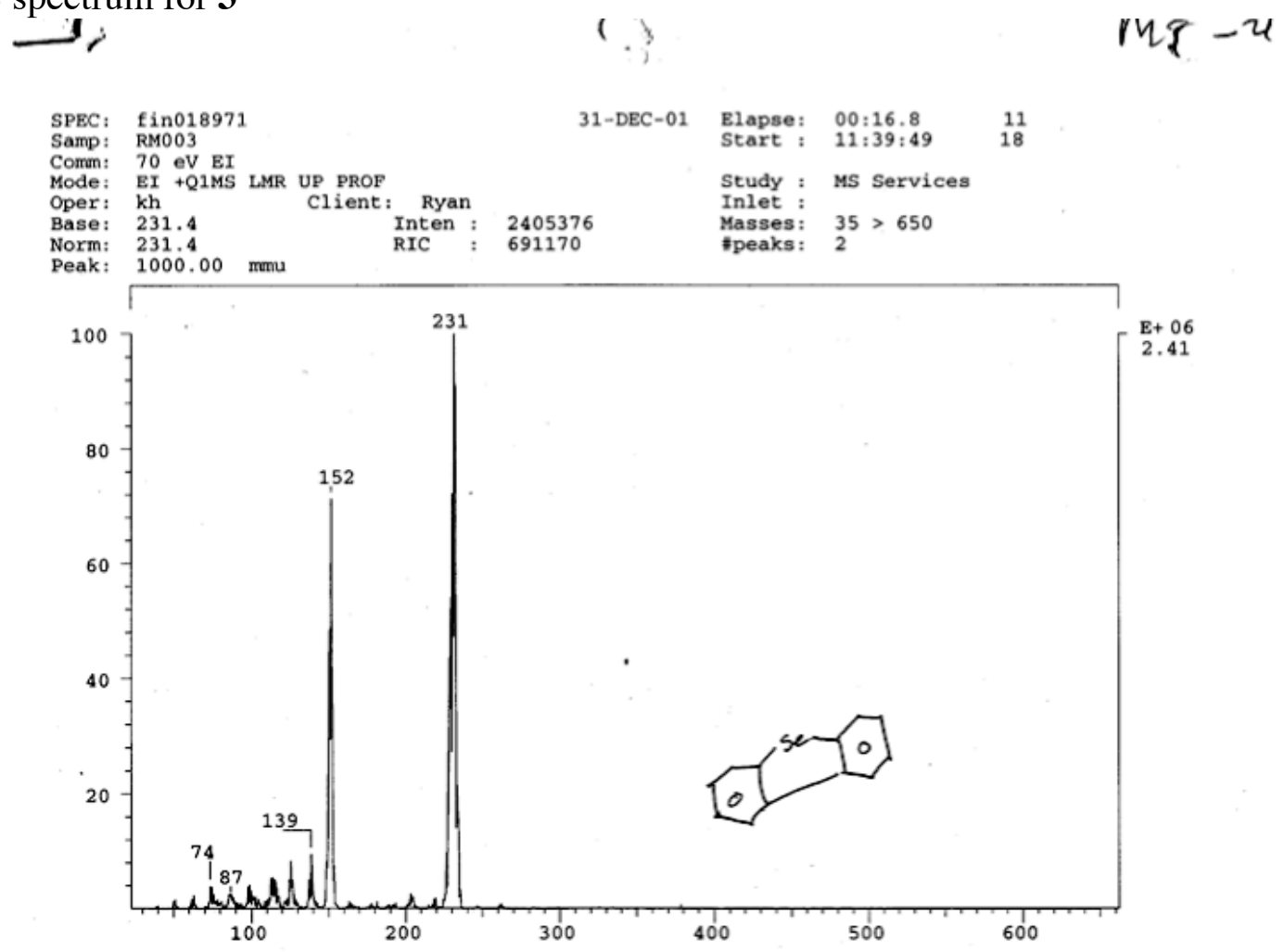


UV spectrum for 3
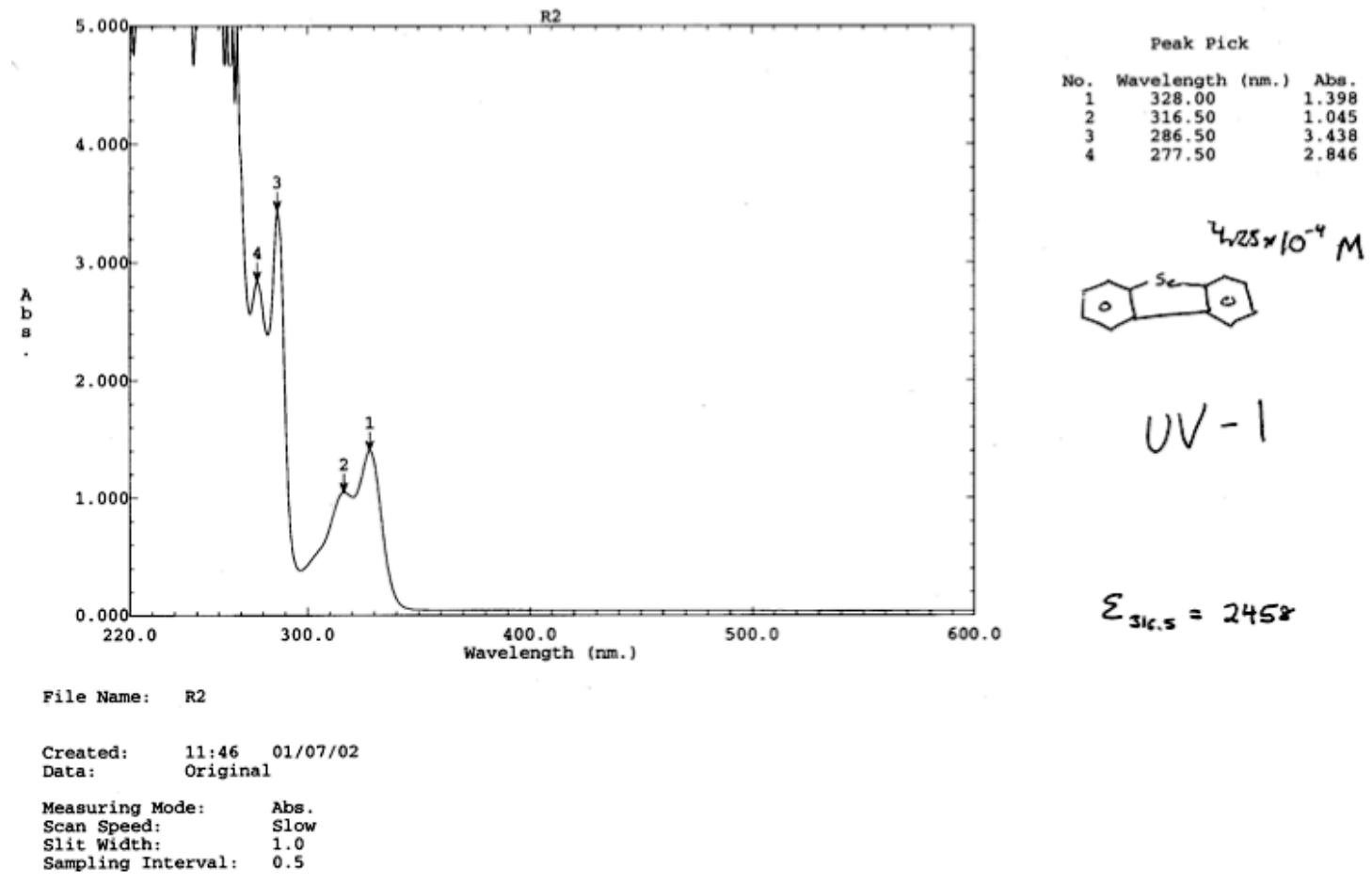
Compound 5

Dibenzoselenophene-Se-Se-dioxide

${ }^{1} \mathrm{H}$ NMR spectrum for 5

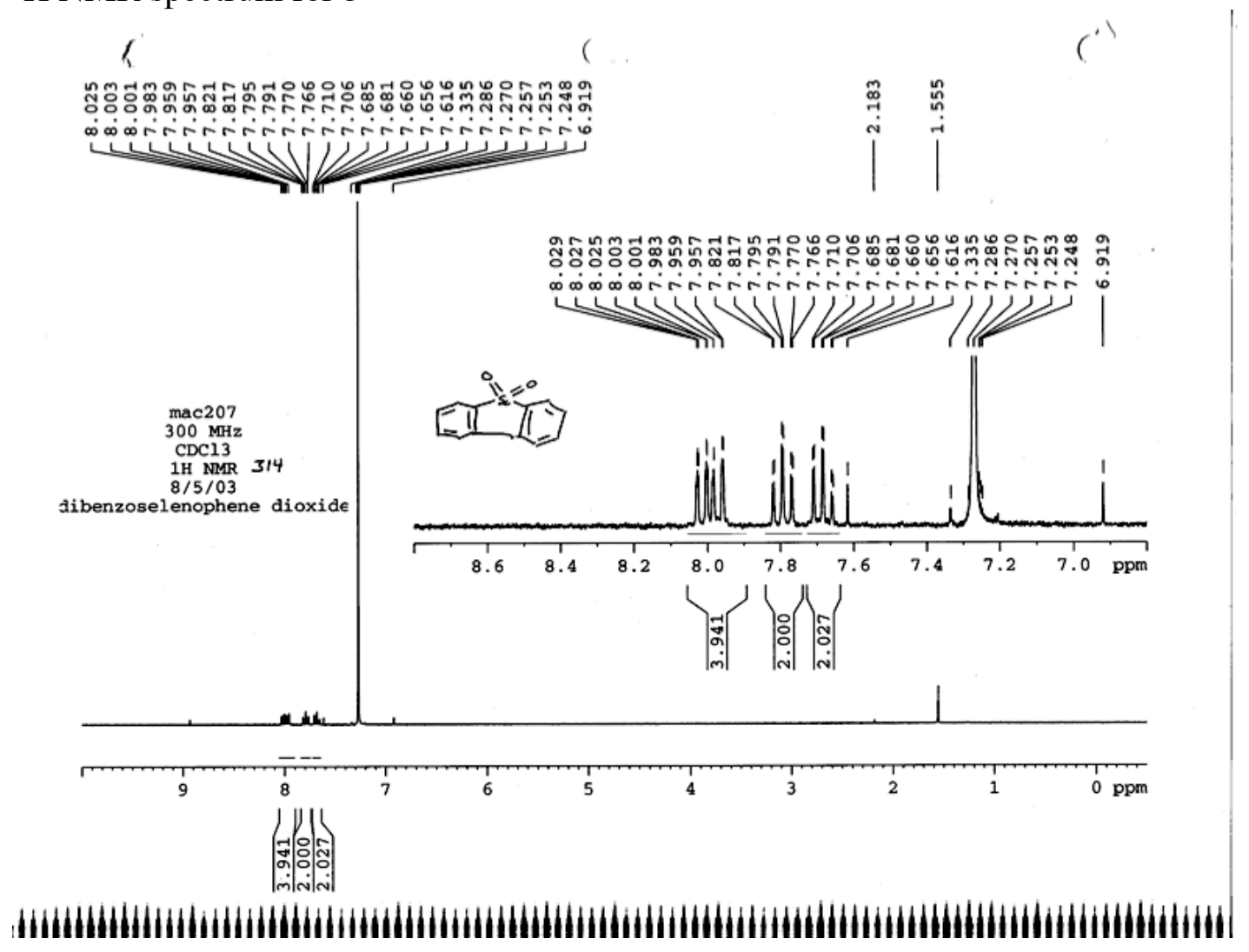


${ }^{13} \mathrm{C}$ NMR spectrum for 5

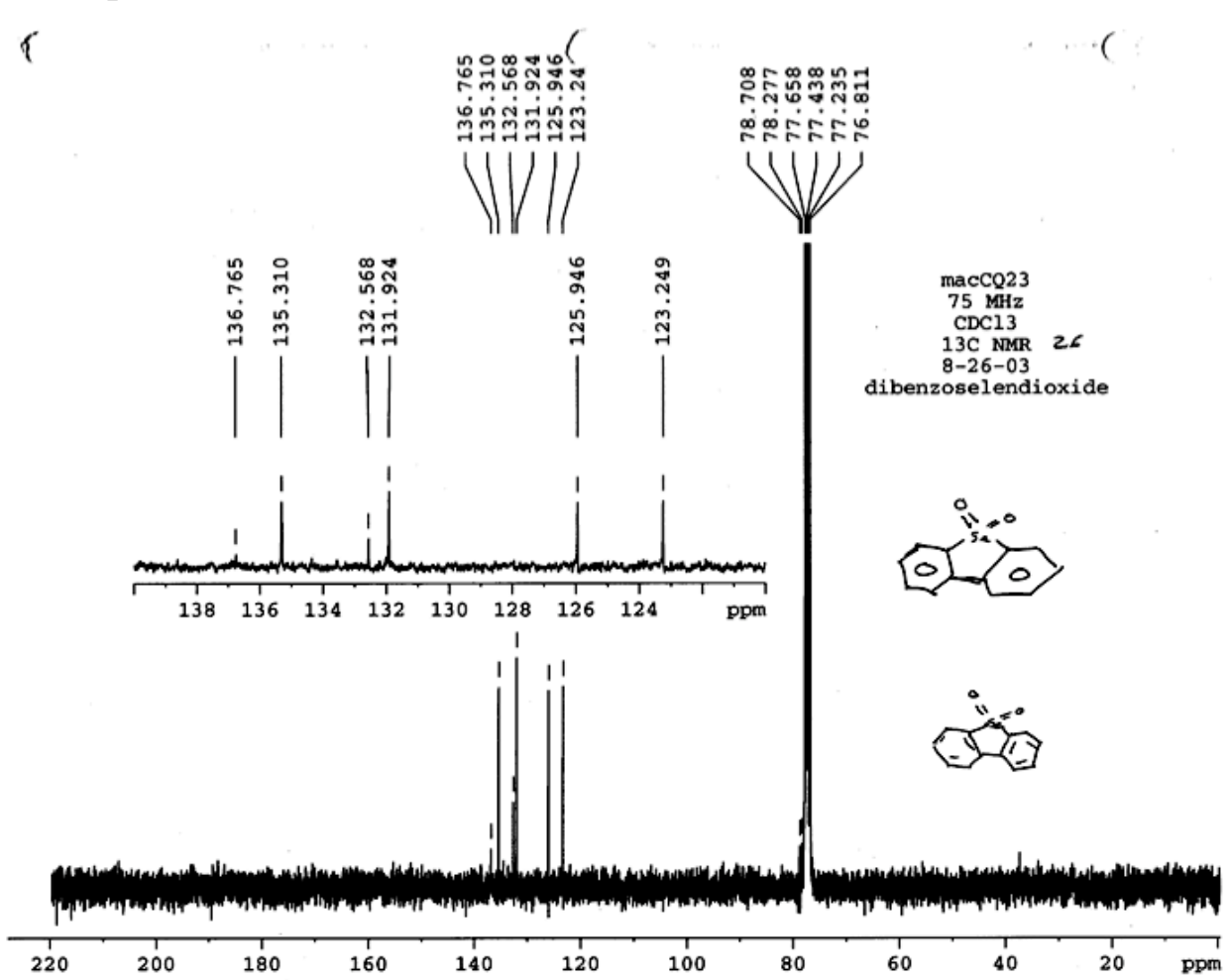

MS spectrum for 5

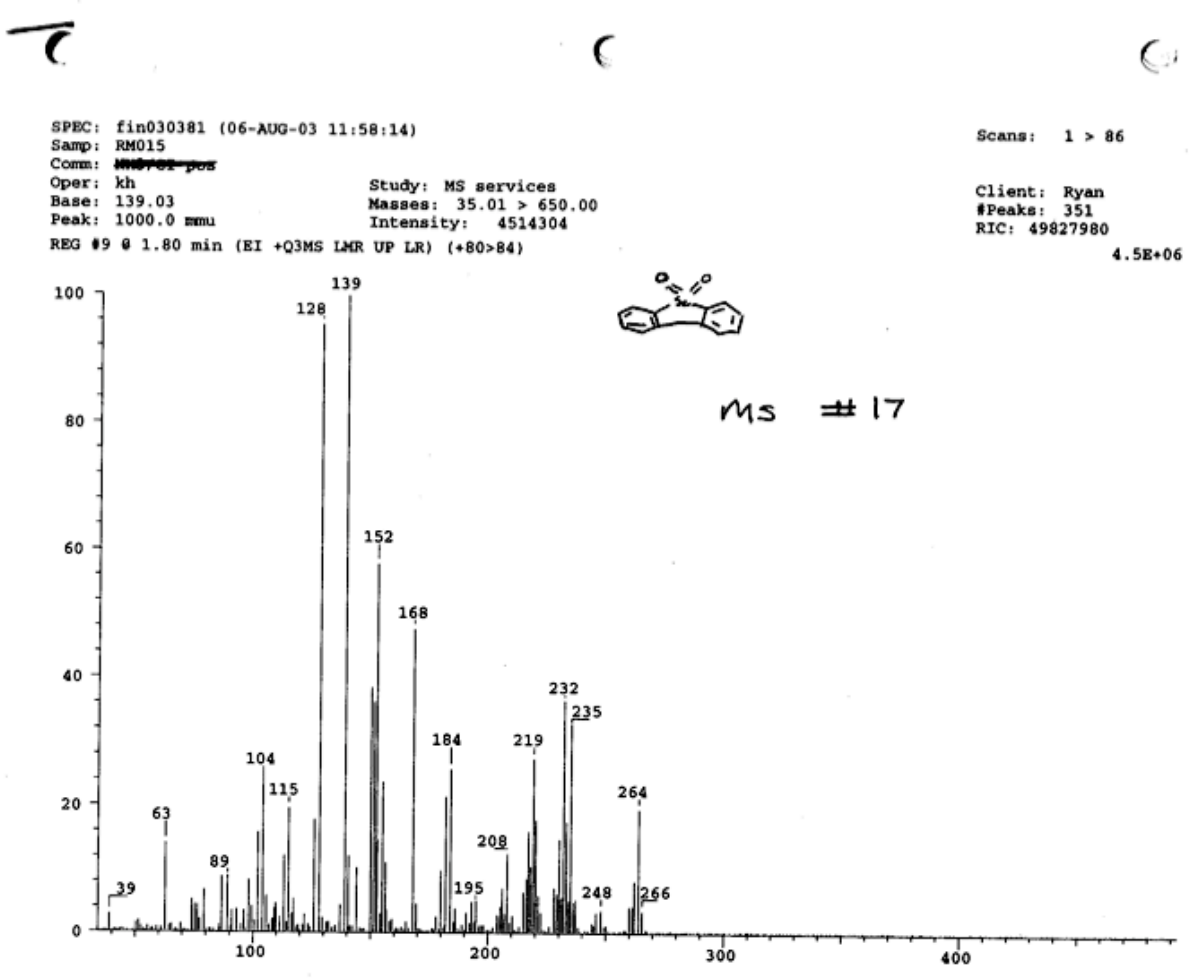

Date: Wed Aug 6 12:00:43 2003 ICIS: 8.3.0 SP2 for OSp1 (V4.0) buila 98-238 from 26-Aug-98 


\section{Compound 8}

10-oxa-9-Selenaphenanthrene-9-oxide

${ }^{1} \mathrm{H}$ NMR spectrum for $\mathbf{8}$

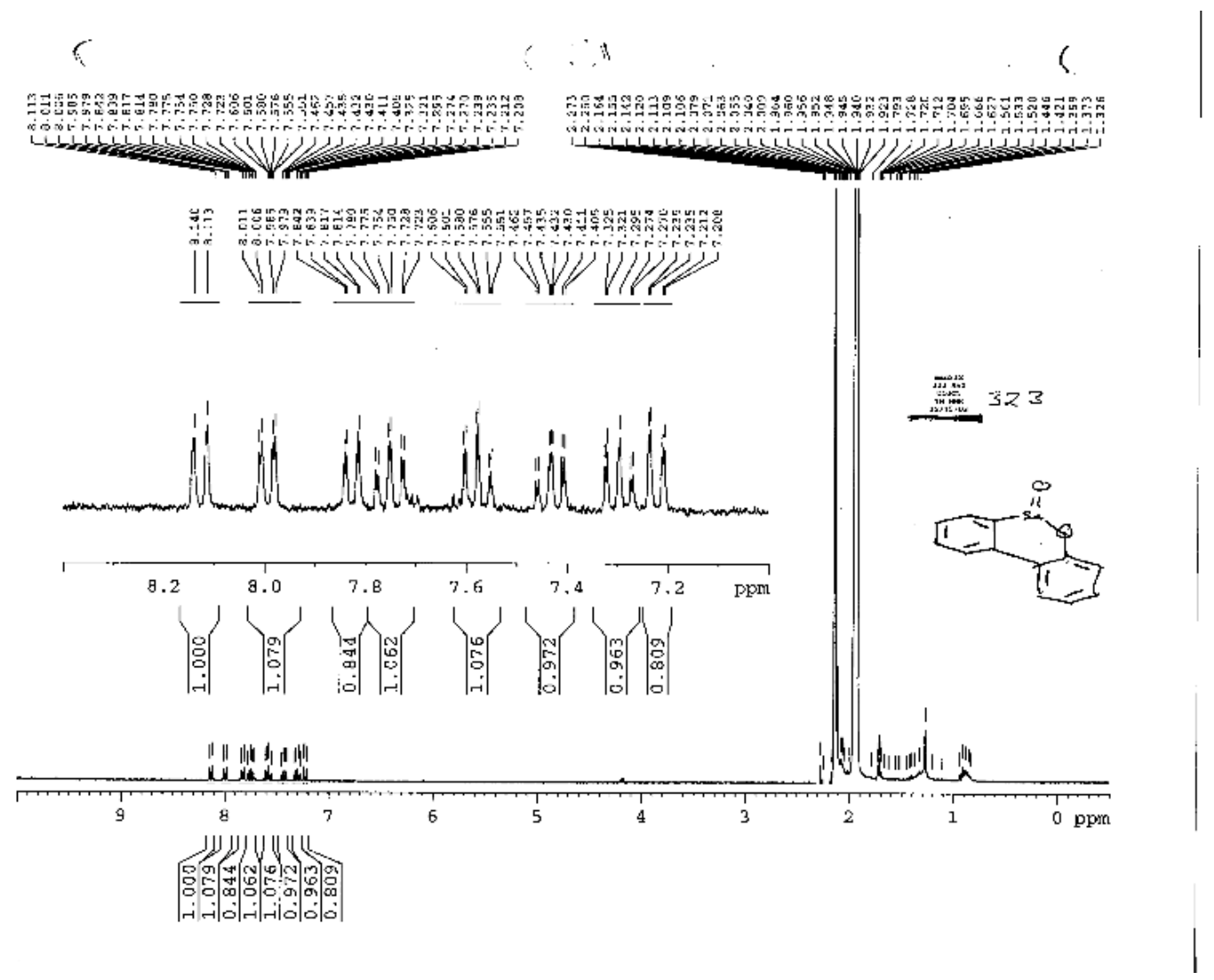




\section{UV spectrum for $\mathbf{8}$}

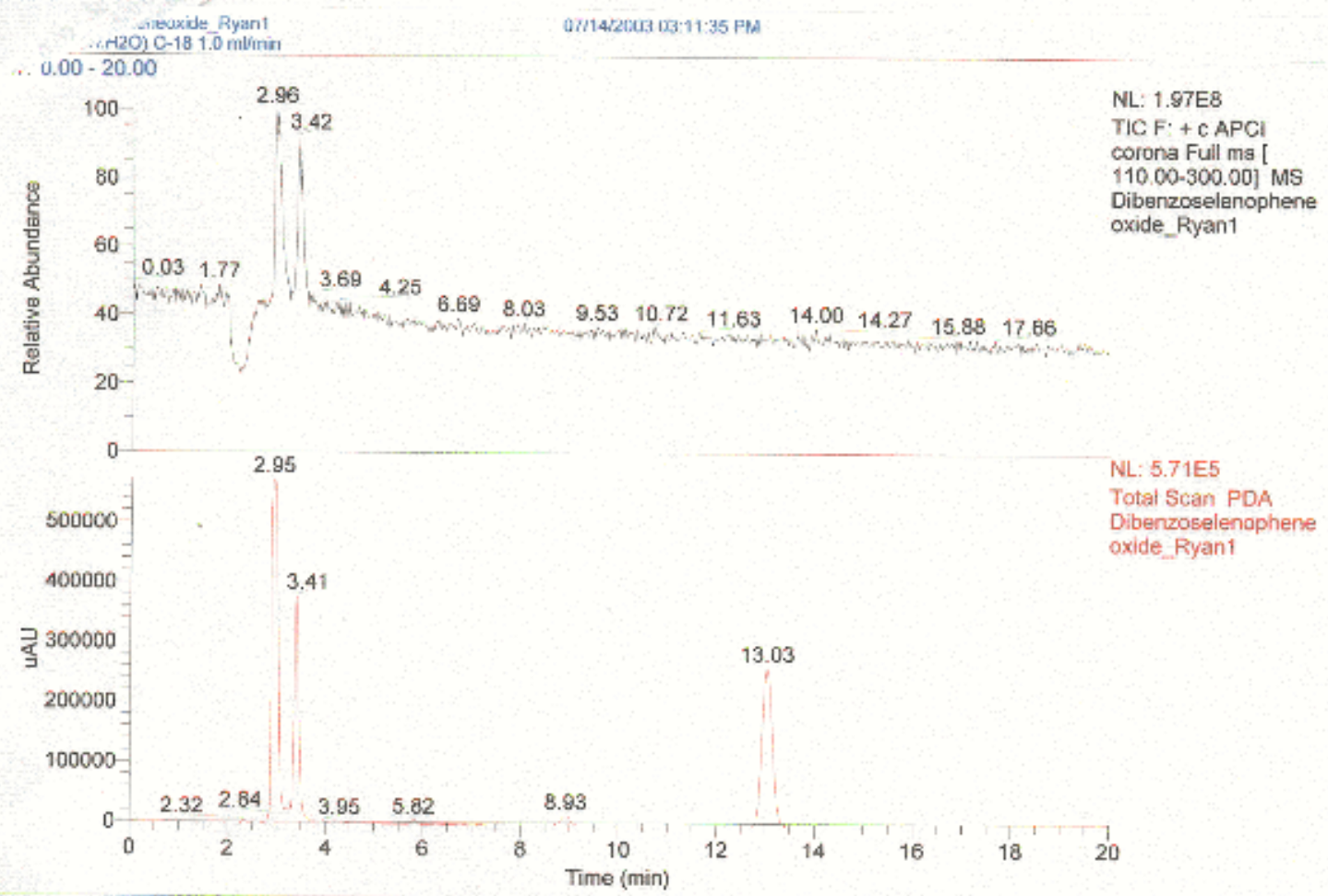

Dibenzoselenapheneaxide_Ryant \#984-1048 RT: $3.28-3.49$ AV: 65 NL-1.65E6 microAU

F: + c APCl corona d Full ms2 264.86@45.00 [ 60.00-540.00]

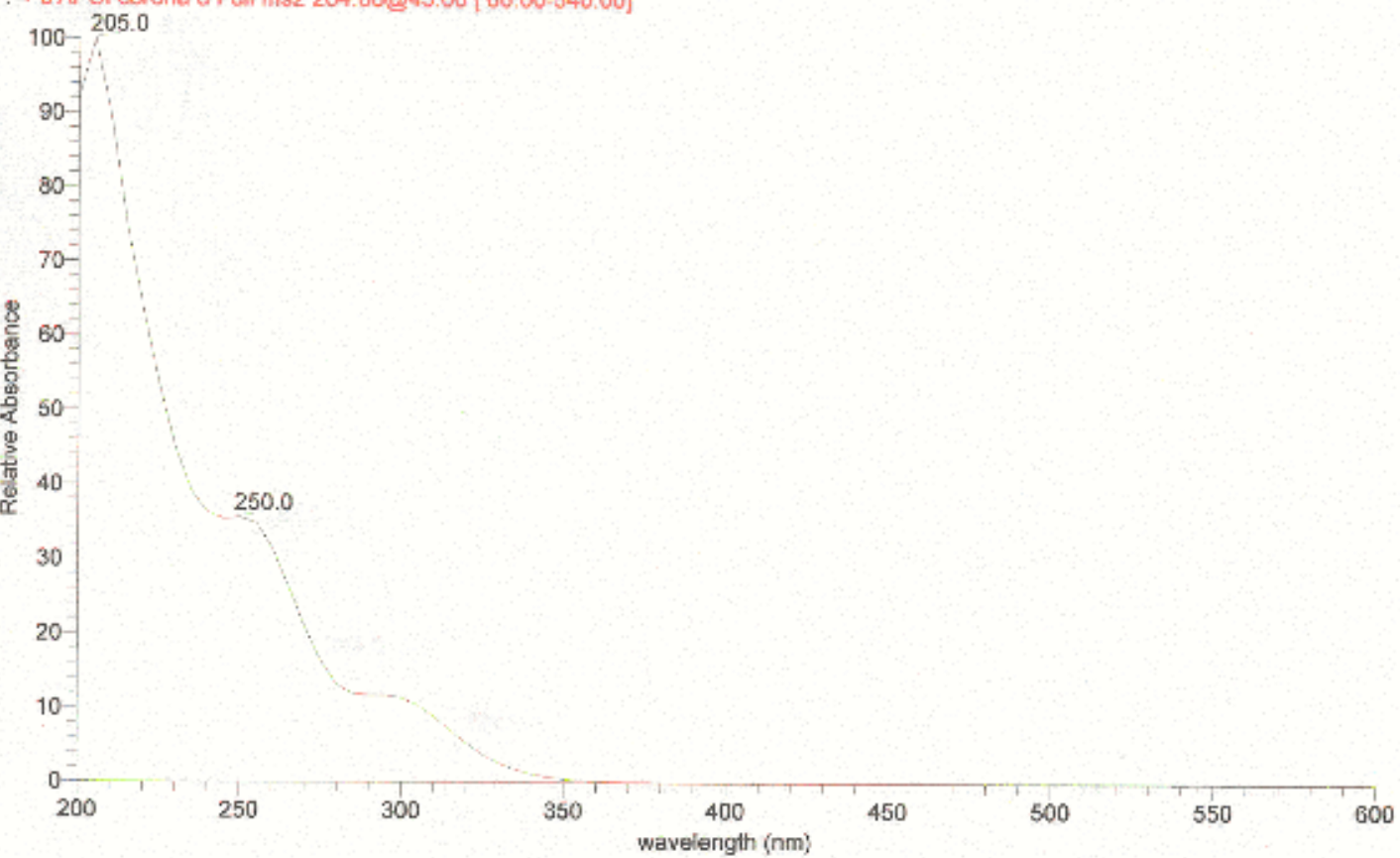




\section{MS spectrum for 8}

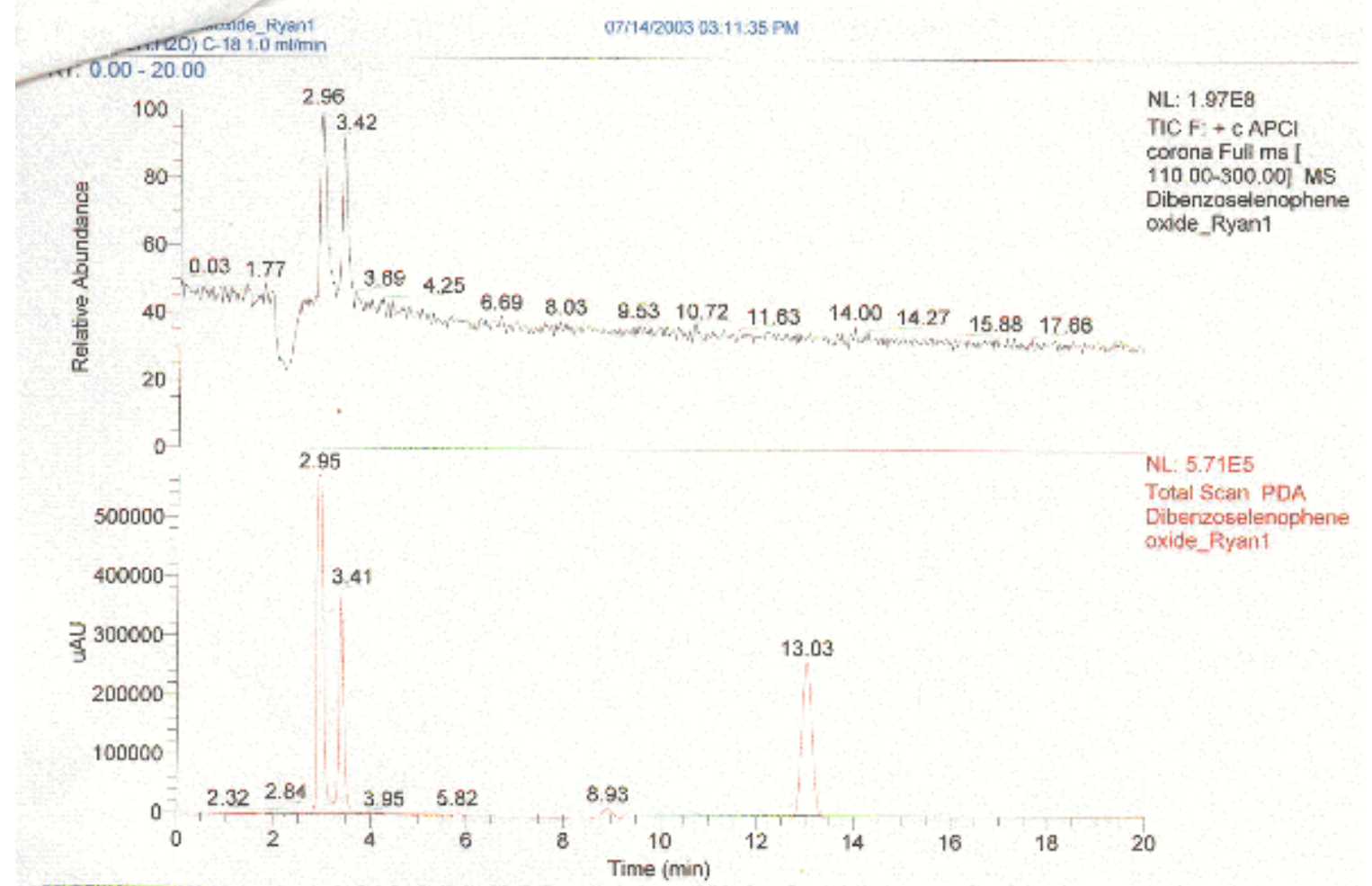

Diberizoselenopheneoxide Ryan1 \#230-256 RT: 3.24-3.54 AV: 13 NL: 1.34E7

$\mathrm{F}:+\mathrm{CAPCl}$ corona Full ms [ 110 00-300.00]

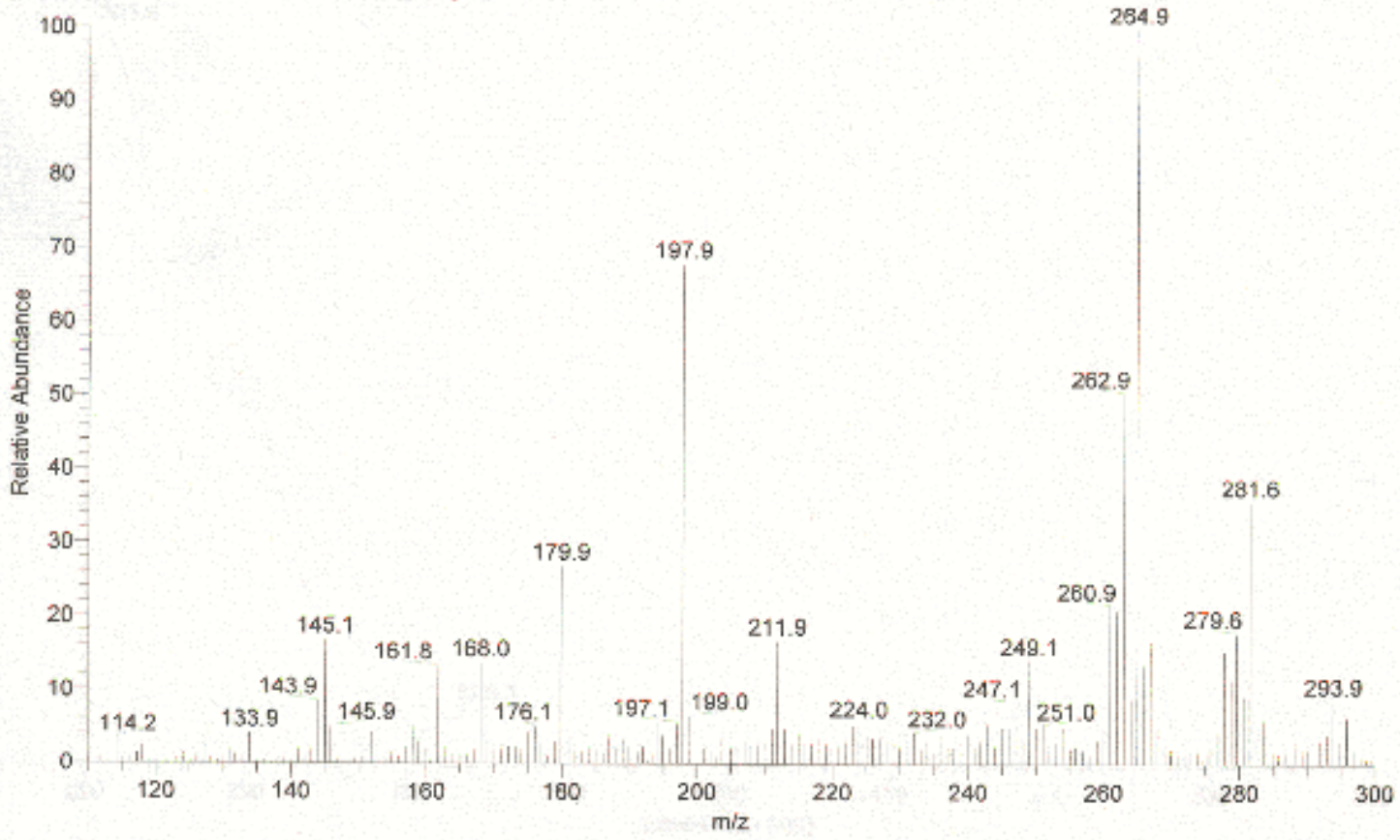




\section{Compound 9}

10-oxa-9-Selenaphenanthrene

${ }^{1} \mathrm{H}$ NMR spectrum for 9

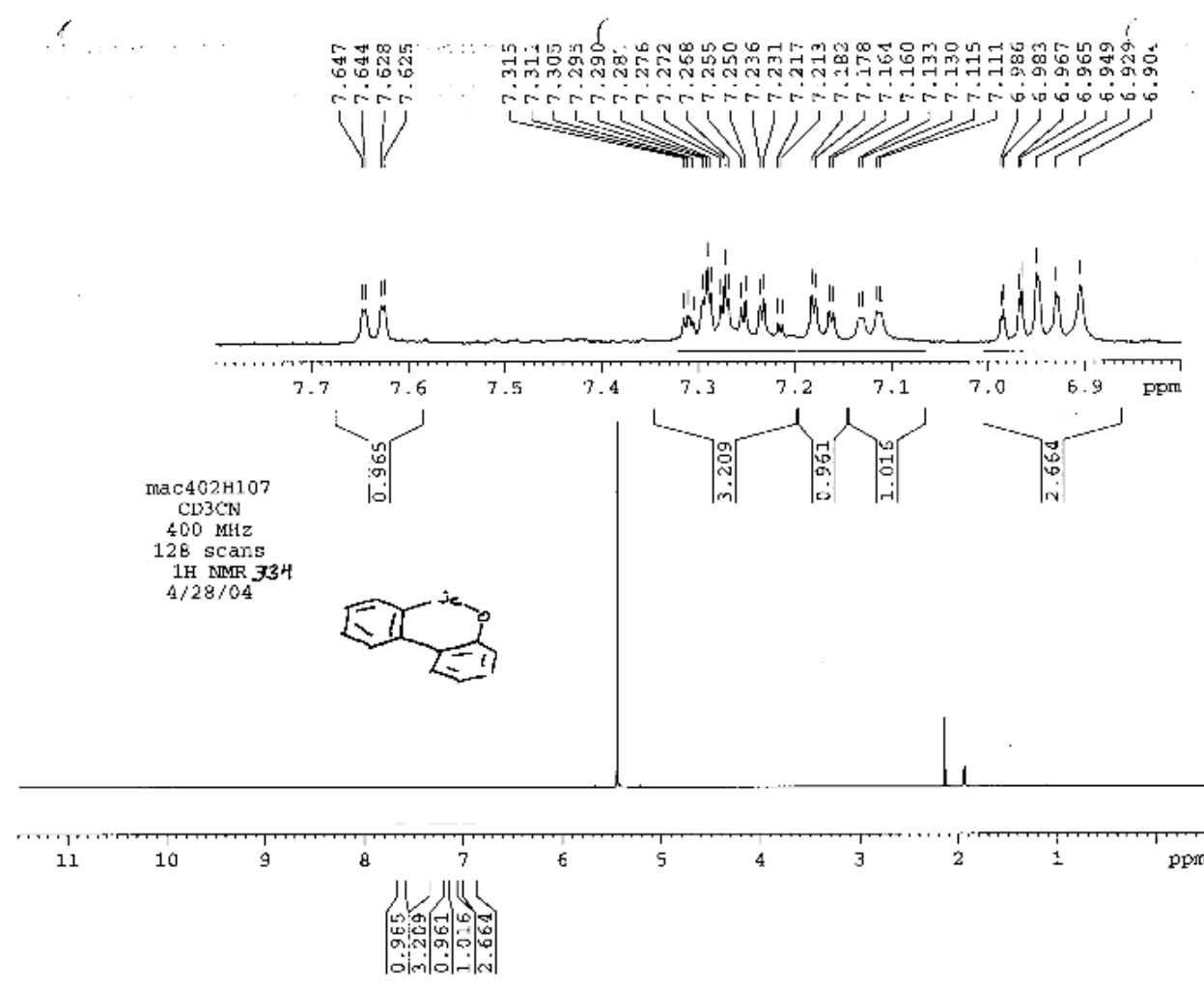




\section{MS spectrum for 9}

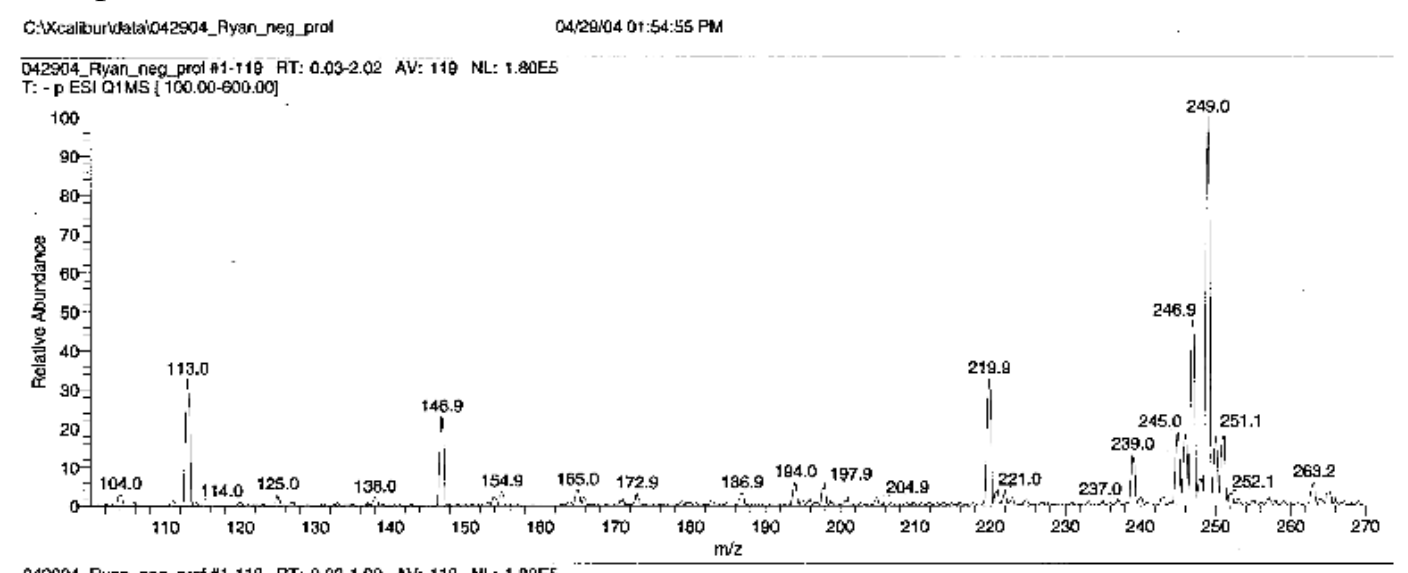

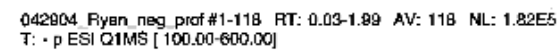

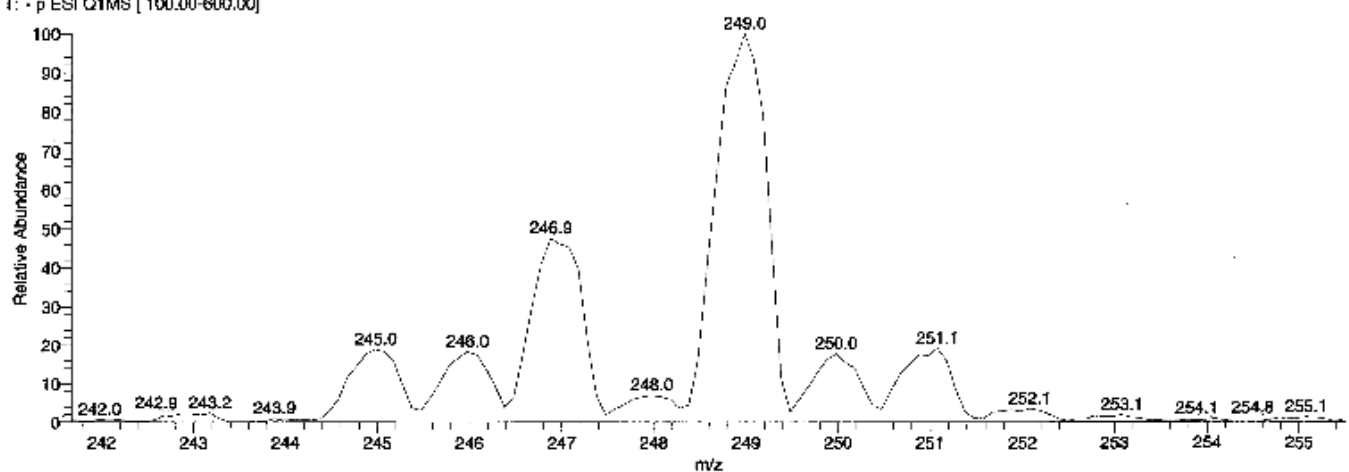

\section{COSY spectrum for 9}

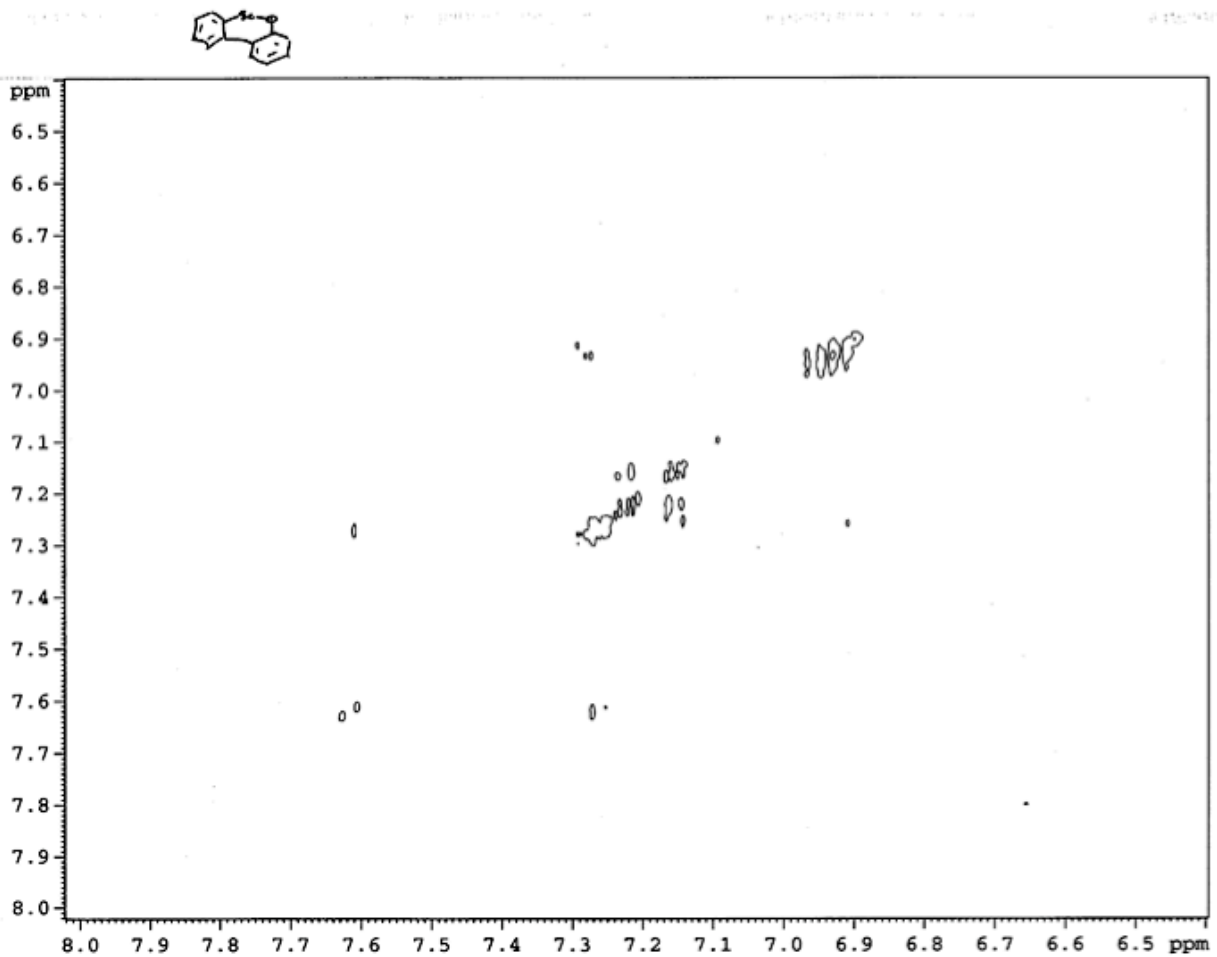

